\title{
NMR Structure-Based Optimization of Staphylococcus aureus Sortase A Pyridazinone Inhibitors
}

\author{
Albert H. Chan ${ }^{1,2,3}$, Sung Wook Yi ${ }^{1}$, Ethan M. Weiner ${ }^{1,2,3}$, Brendan R. Amer ${ }^{1,2,3}$, \\ Christopher K. Sue ${ }^{1}$, Jeff Wereszczynski ${ }^{4}$, Carly A. Dillen ${ }^{8}$, Silvia Senese ${ }^{1}$, Jorge Z. \\ Torres $^{1}$, J. Andrew McCammon ${ }^{5,6,7}$, Lloyd S. Miller ${ }^{8}$, Michael E. Jung ${ }^{1,{ }^{*}}$, and Robert T. \\ Clubb $^{1,2,3, *}$ \\ ${ }^{1}$ Department of Chemistry and Biochemistry, University of California, Los Angeles, 611 Charles \\ Young Drive East, Los Angeles, CA 90095, USA \\ ${ }^{2}$ UCLA-DOE Institute of Genomics and Proteomics, University of California, Los Angeles, 611 \\ Charles Young Drive East, Los Angeles, CA 90095, USA \\ ${ }^{3}$ Molecular Biology Institute, University of California, Los Angeles, 611 Charles Young Drive East, \\ Los Angeles, CA 90095, USA \\ ${ }^{4}$ Department of Physics and Center for Molecular Study of Condensed Soft Matter, Illinois \\ Institute of Technology, Chicago, IL 60616, USA \\ ${ }^{5}$ Department of Chemistry and Biochemistry, University of California, San Diego, La Jolla, CA \\ 92093, USA \\ ${ }^{6}$ Howard Hughes Medical Institute, University of California, San Diego, La Jolla, CA 92093, USA \\ ${ }^{7}$ Department of Pharmacology, University of California, San Diego, La Jolla, CA 92093, USA \\ ${ }^{8}$ Department of Dermatology, Johns Hopkins University School of Medicine, Baltimore, MD \\ 21231, USA
}

\section{Abstract}

Staphylococcus aureus is a leading cause of hospital-acquired infections in the United States and is a major health concern as methicillin-resistant $S$. aureus (MRSA) and other antibiotic resistant strains are common. Compounds that inhibit the $S$. aureus sortase (SrtA) cysteine transpeptidase may function as potent anti-infective agents as this enzyme attaches virulence factors to the bacterial cell wall. While a variety of SrtA inhibitors have been discovered, the vast majority of these small molecules have not been optimized using structure-based approaches. Here we have used NMR spectroscopy to determine the molecular basis through which pyridazinone-based

\footnotetext{
*To whom correspondence should be addressed: Dr. Robert T. Clubb, Dept. of Chemistry and Biochemistry, University of California, Los Angeles, 602 Boyer Hall, Los Angeles, CA 90095. Tel.: 310-206-2334; rclubb@ mbi.ucla.edu; Dr. Michael E. Jung, Dept. of Chemistry and Biochemistry, University of California, Los Angeles, 3505A Molecular Sciences Building, Los Angeles, CA 90095. Tel.: 310-825-7954; jung@ chem.ucla.edu.

Conflict of Interest

There are no conflicts of interest.

Supporting Information

Overlay of the in vitro dose-response curves of 2-10, 2-17, 2-54 and 2-62 (Fig. S1); synthetic procedures of the pyridazinone analogues.
} 
small molecules inhibit SrtA. These inhibitors covalently modify the active cysteine thiol and partially mimic the natural substrate of SrtA by inducing the closure of an active site loop. Computational and synthetic chemistry methods led to second generation analogs that are $\sim 70$-fold more potent than the lead molecule. These optimized molecules exhibit broad-spectrum activity against other types of class A sortases, have reduced cytotoxicity and impair SrtA-mediated protein display on $S$. aureus cell surface. Our work shows that pyridazinone analogs are attractive candidates for further development into anti-infective agents, and highlights the utility of employing NMR spectroscopy and solubility-optimized small molecules in structure-based drug discovery.

\section{Graphical abstract}

NMR spectroscopy was used to determine the structure of Staphylococcus aureus sortase A transpeptidase enzyme in complex with a pyridazinone-based small molecule, a potential antiinfective agent. Computational and synthetic chemistry methods led to second generation analogs that are 70-fold more potent than the lead molecule, less cytotoxic and effective at impairing sortase A-mediated protein display on the surface of $S$. aureus. These pyridazinone analogs are attractive candidates for further development into anti-infective agents.

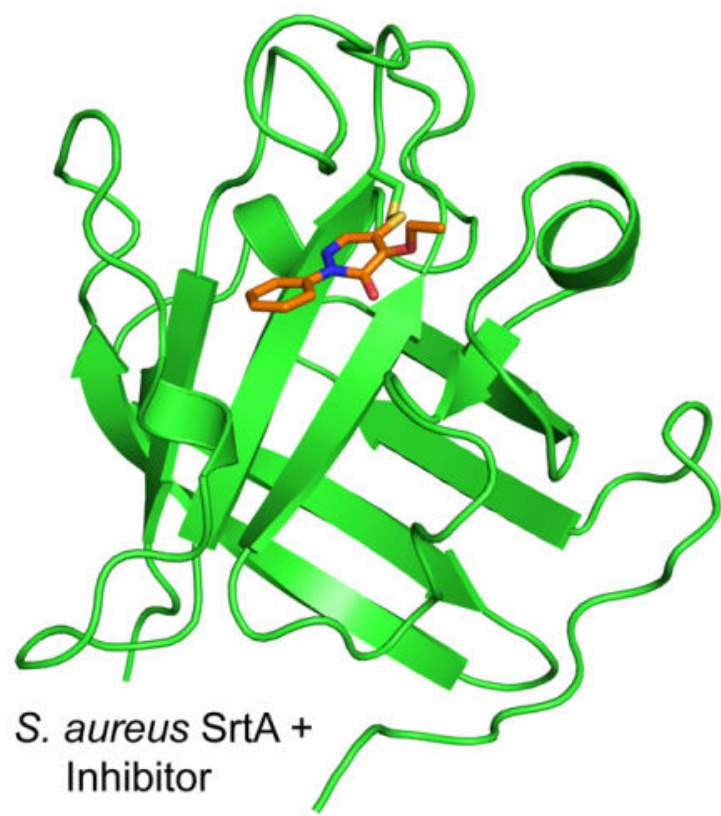

\section{Keywords}

Sortase; SrtA; transpeptidase; protein-inhibitor complex; NMR; molecular dynamics; molecular docking; protein structure; Staphylococcus aureus

\section{Introduction}

Staphylococcus aureus is a leading cause of hospital- and community-acquired infections in the United States. This bacterial pathogen is estimated to cause 11,000 deaths annually in 
the United States. It inflicts a wide range of life-threatening diseases such as pneumonia, meningitis, osteomyelitis, endocarditis, toxic shock syndrome, bacteremia, and sepsis (1) a . $S$. aureus skin and soft tissue infections also represent a major clinical problem as they result in over 11 million outpatient and emergency room visits, and close to 500,000 hospital admissions per year in the U.S. (2,3). The rise of methicillin-resistant $S$. aureus (MRSA) is a major health problem that has created a pressing need for new antibiotics. MRSA harbors genes that confer resistance to $\beta$-lactam antibiotics and is now endemic in hospitals. $S$. aureus has also developed resistance to other antibiotics, including last resort and newer generation drugs, such as vancomycin, daptomycin and linezolid (4-6). The $S$. aureus sortase A (SrtA) enzyme is an attractive molecular target for the development of novel antibiotics. This is because this enzyme covalently attaches a range of protein virulence factors to the surface of $S$. aureus that play critical roles in the infection process, including promoting bacterial adhesion to host tissues, acquisition of essential nutrients, and the evasion and suppression of the immune response $(7,8)$. Several studies have shown that $s r t A^{-} S$. aureus mutants have reduced virulence in animal models of infection, presumably because their surfaces are devoid of key protein factors required to colonize host tissue and evade the immune response $(9,10)$. SrtA-related enzymes are also used by other clinically important pathogens to display factors that are required for their virulence (e.g.

Enterococcus faecilis, Listeria monocytogenes, Bacillus anthracis, Streptococcus pyogenes and Streptococcus pneumoniae) (10). Thus, small molecule SrtA enzyme inhibitors could function as potent anti-infective agents to treat MRSA and infections caused by other types of Gram-positive bacteria.

SrtA resides on the extracellular membrane where it covalently attaches proteins to peptidoglycan by catalyzing a transpeptidation that joins proteins containing a C-terminal cell wall sorting signal (CWSS) to the cross-bridge peptide (11). Besides its important role in pathogenesis, SrtA has several other properties that make it an attractive drug target: (i) it has no human homolog, reducing the likelihood of off-target effects, (ii) it is located on the bacterial surface such that inhibitors do not need to cross the cell membrane thereby reducing potential cytotoxicity, and (iii) it is not required for the growth of $S$. aureus and other clinically important microbes when they are outside their human host (12). Therefore, SrtA inhibitors that selectively target virulence mechanisms could have a distinct advantage over conventional antibiotics, as they may not induce the same selective pressures that lead to drug resistance $(13,14)$.

Several research groups have sought to identify small molecule SrtA inhibitors that could be developed into therapeutics $(10,15)$. The small molecules that have thus far been identified include, natural products and synthetic molecules identified by screening compound libraries, and rationally designed molecules that mimic the substrate or transition state intermediates (10). In addition, virtual screening methods have been employed to identify inhibitors using the structure of the apo- or substrate-bound forms of the enzyme (10). However, a clinically useful SrtA inhibitor has yet to be developed. A major obstacle

anote

Center for Disease Control and Prevention. 2013. Antibiotic Resistance Threats in the United States, 2013. http://www.cdc.gov/ drugresistance/threat-report-2013/ 
hindering drug development has been the difficulty in applying structure-based methods to optimize SrtA-inhibitors. This is because the active site of SrtA is structurally disordered in its apo-state making it difficult to model drug-enzyme interactions computationally and potentially hindering the application of x-ray crystallography that require crystallization of the SrtA-inhibitor complex. At present, only Zhulenkov and colleagues have used NMR spectroscopy to visualize how SrtA binds to an inhibitor, a benzisothiazolinone-based small molecule that irreversibly modifies the enzyme (16). However, the structure of the inhibitorSrtA complex was determined at low resolution. Maresso, et al. have also structurally characterized covalent inhibitors of the aryl ( $\beta$-amino)ethyl ketone class by co-crystallizing the inhibitors with the sortase B from Bacillus anthracis (Ba-SrtB) (17). However, Ba-SrtB may not be a good platform from which to optimize $S$. aureus SrtA inhibitors, as SrtA and Ba-SrtB have distinct active site structures and recognize different sorting signal substrates; SrtA and Ba-SrtA recognize LPXTG and NPQTN sorting signals, respectively (18). Thus, while many compounds inhibit the activity of SrtA in vitro, a lack of structural data to rationally optimize these molecules for clinical applications has been problematic.

Using high throughput screening (HTS) methods we previously identified pyridazinonebased molecules that are potent inhibitors of SrtA (19). However, these molecules were not optimized using structure-based approaches because they were poorly soluble in water, which made it difficult to experimentally determine the three-dimensional structure of the enzyme-inhibitor complex, and because conformational disorder in the SrtA's active site made it difficult to computationally model enzyme-inhibitor interactions. We have now overcome this problem by using a highly soluble pyridazinone analogue whose complex with SrtA could be determined using NMR spectroscopy. We show that pyridazinone inhibitors partially mimic the natural substrate, by inducing a conformational change in the active site that incompletely closes a key active site loop, resulting in a final structure that is reminiscent of both the apo- and sorting signal bound forms of the enzyme. Guided by computational studies of the complex, a series of pyridazinone analogues were synthesized and their efficacy evaluated in vitro and in cell culture. This work led to the discovery of 2(3-fluorophenyl)-4-(3-hydroxypropoxy)-5-mercaptopyridazin-3(2H)-one, which inhibits SrtA mediated protein display in $S$. aureus and improves inhibitory activity over the lead molecule 70 -fold ( $\mathrm{IC}_{50}$ value of $21 \pm 14 \mathrm{nM}$ ). The use of a solubility optimized inhibitor analogue for NMR studies of the complex was critical and may be a generally useful strategy to study other enzyme inhibitor interactions.

\section{Materials and Methods}

\section{Preparation of the SrtA-inhibitor complex for NMR and mass spectrometry studies}

The catalytic domain of SrtA (residues $\mathrm{Gln}^{60}$-Lys ${ }^{206}$ ) was prepared as described previously (20). To form a SrtA:inhibitor complex, $0.5 \mathrm{mM}$ uniformly ${ }^{15} \mathrm{~N}$ - and ${ }^{13} \mathrm{C}$-labeled SrtA was incubated with $5 \mathrm{mM}$ of sodium thiolate 4-ethoxy-5-mercapto-2-phenylpyridazin-3(2H)-one (hereafter referred as 2-salt) in $50 \mathrm{mM}$ Tris, pH 6.4, $150 \mathrm{mM} \mathrm{NaCl}, 20 \mathrm{mM} \mathrm{CaCl}_{2}$ for $72 \mathrm{~h}$ at room temperature. The complex was then dialyzed into NMR buffer that consisted of 50 $\mathrm{mM}$ Tris, $\mathrm{pH}$ 6.4, $150 \mathrm{mM} \mathrm{NaCl}$, and $20 \mathrm{mM} \mathrm{CaCl}_{2}$. Two NMR samples were studied that each contained $1.5 \mathrm{mM}$ SrtA-inhibitor complex dissolved NMR buffer and either $7 \%$ or 
99.999\% $\mathrm{D}_{2} \mathrm{O}$. Mass spectrometry was used to verify that the inhibitor forms a disulfide bond with Cys ${ }^{184}$ in SrtA. The SrtA:2-salt complex was first digested with trypsin by incubating $20 \mu \mathrm{M}^{15} \mathrm{~N}^{13} \mathrm{C}$-SrtA:2-salt complex with $5 \mu \mathrm{g} / \mathrm{mL}$ trypsin for $24 \mathrm{~h}$ at $37^{\circ} \mathrm{C}$. The digestion reaction mixture was then split into two aliquots. To one of the aliquots, DTT was added to a final concentration of $5 \mathrm{mM}$. Both aliquots were then subjected to LC-MS analysis to monitor the masses of the cleaved peptides. LC-MS experiments were carried out on a Waters Acquity UPLC connected to a Waters LCT-Premier XE Time of Flight Instrument controlled by MassLynx 4.1 software (Waters Corp., Milford, MA, USA). Digested protein samples in $20 \mathrm{mM}$ HEPES, $5 \mathrm{mM} \mathrm{CaCl}_{2}$ were separated using an Phenomenex Hydro-RP column $(3.0 \times 50 \mathrm{~mm}, 4 \mu \mathrm{m}$ packing $)$ and were eluted with a gradient of $2-50 \%$ solvent $\mathrm{B}$ over $10 \mathrm{~min}$ using a flow rate of $0.425 \mathrm{~mL} / \mathrm{min}$ (solvent $\mathrm{A}$ : water, solvent B: acetonitrile, both with $0.3 \%$ formic acid). The mass spectrometer was equipped with a Multi-Mode Source operated in the electrospray mode. Mass spectra were recorded from a mass of $70-2,000$ Daltons. Capillary voltage was set to $1700 \mathrm{~V}$ and the source/desolvation gas temperatures were $120^{\circ} \mathrm{C} / 350^{\circ} \mathrm{C}$, respectively. The ion abundance values for product ions at m/z 819.5, 942.5, 1638 and 1886 were monitored by generating extracted ion chromatograms using a $0.5 \mathrm{Da}$ mass window and integrating the peaks of interest. These ions had retention time values of 5.09, 6.86, 5.09 and $6.86 \mathrm{~min}$, respectively.

\section{NMR spectroscopy and structure determination}

NMR spectra of the SrtA:inhibitor complex were acquired at 298 K on Bruker Avance 500-, 600-, and 800-MHz spectrometers equipped with triple resonance cryogenic probes. NMR spectra were processed using NMRPipe (21) and analyzed using the PIPP (22) and CARA (version 1.8.4) (23) software packages. Chemical shift assignments $\left({ }^{1} \mathrm{H},{ }^{13} \mathrm{C},{ }^{15} \mathrm{~N}\right)$ of SrtA were obtained by analyzing the following experiments: HNCA, HNCACB, CBCA(CO)NH, HNCO, HN(CA)CO, ${ }^{15}$ N-edited TOCSY, HNHA, HNHB, HBHA(CO)NH, HCCH-TOCSY, $\mathrm{HCCH}-\mathrm{COSY}$, (HB)CB(CGCDCE)HE, and (HB)CB(CGCD)HD (reviewed in $(24,25)$ ). Chemical shift assignments for the inhibitor were obtained by analyzing a two-dimensional (F2) ${ }^{13} \mathrm{C}$-filtered NOESY spectrum. The majority of $\phi$ and $\psi$ dihedral angle restraints were obtained using the program TALOS+ (26). Additional backbone $\phi$ angle restraints were obtained by analyzing HNHA spectra (27). Intramolecular protein distance restraints were obtained from three-dimensional ${ }^{15} \mathrm{~N}$ - and ${ }^{13} \mathrm{C}$-edited NOESY spectra. Intermolecular distance restraints were obtained by analyzing two-dimensional (F2) ${ }^{13} \mathrm{C}$-filtered NOESY and ${ }^{13} \mathrm{C}$-edited NOESY-HSQC spectra of the complex.

NOE assignments were initially obtained automatically using the programs ATNOS/ CANDID controlled by UNIO $(28,29)$ utilizing XPLOR-NIH (version 2.23) (30). NOE assignments were then verified manually for the active site residues ( $\mathrm{Val}^{161}-\mathrm{Asp}^{175}[\beta 6 / \beta 7$ loop], Thr ${ }^{183}$-Lys ${ }^{196}$ [ $\beta 7 / \beta 8$ loop], and side chains of Leu ${ }^{97}, \mathrm{Ser}^{116}$, His ${ }^{120}$, $\mathrm{Thr}^{180}$, $\mathrm{Ile}^{182}$, and $\mathrm{Val}^{201}$ ) by inspecting the NOESY data. During inspection of the NOESY data, additional NOE restraints involving active site residues were identified and included in subsequent structure calculations. Final structures were calculated using XPLOR-NIH based on the lowest energy conformer of the previously solved apo-SrtA NMR structure (PDB 1IJA) (20). During the course of the structure calculations, backbone and side chain atoms of active site residues Val ${ }^{161}$-Asp ${ }^{175}$ ( $\beta 6 / \beta 7$ loop) and $\mathrm{Thr}^{183}$-Lys $^{196}$ ( $\beta 7 / \beta 8$ loop), as well as 
side chain atoms of $\mathrm{Leu}^{97}, \mathrm{Ser}^{116}$, $\mathrm{His}^{120}, \mathrm{Thr}^{180}$, Ile ${ }^{182}, \mathrm{Arg}^{197}, \mathrm{Il}^{199}$, and $\mathrm{Val}^{201}$, were allowed to move while all other SrtA atoms were held fixed in space. Residues within the $\beta 6 / \beta 7$ and $\beta 7 / \beta 8$ loops were allowed to move because they form the surface surrounding the presumed inhibitor binding site, and previous crystal and NMR structural studies of apo and substrate (LPAT*) bound SrtA reveal these loops adopt distinct conformations as a result of substrate binding $(20,31,32)$. Mobility of other active site residues was restricted to their side chains because while they make contacts with the sorting signal substrate in the SrtA:LPAT* structure, they do not demonstrate significant backbone rearrangement. Nonactive site residues were kept rigid because they show little difference $(\mathrm{RMSD}=0.8 \AA$ ) between the apo and substrate bound SrtA structures, and such structural information is of little value for structure-based drug design of a competitive inhibitor. In the final set of calculations, a thiol group within the inhibitor was attached to $\mathrm{Cys}^{184}$ through a disulfide bond. A single hydrogen bond between the backbone carbonyl of Gly ${ }^{167}$ and the backbone amide of $\mathrm{Asp}^{170}$ was also used to stabilize the $33_{10}$-helix within the $\beta 6 / \beta 7$ loop, and was substantiated by characteristic NOE patterns from Gly ${ }^{167}$-Asp ${ }^{170}$. A total of 50 structures were calculated, of which 46 had no NOE, dihedral angle, or scalar coupling violations greater than $0.5 \AA, 5^{\circ}$, or $2 \mathrm{~Hz}$, respectively. Of these, 20 structures with the lowest overall energy were chosen to represent the structure of the SrtA-inhibitor complex. The programs MOLMOL (33) and PyMOL (34) were used to generate figures.

\section{In silico screening of pyridazinone analogues}

Ligand preparation, receptor preparation, grid generation and docking were all conducted with Schrödinger Suite 2011 (Schrödinger LLC, New York, NY, USA). Derivatives of 4ethoxy-5-mercapto-2-phenylpyridazin-3(2H)-one for docking experiments were generated with the CombiGlide application. In CombiGlide, 5-mercapto-2-phenylpyridazin-3(2H)-one was defined as the core, and 225, 104, 104, 104, 32, 32, and 22 substituents were created at R2, R3, R4, R5, R6, R7, and R8 sites, respectively (see Fig. 4 for definition of the R sites). Substituents were chosen based on the NMR structure of the complex (e.g. polar substituents were added to sites on the inhibitor scaffold that projected into the solvent). The substituents at the R2 site included various polar and non-polar groups of different sizes, as well as a series of substituents with the general formula $-\mathrm{O}\left(\mathrm{CH}_{2}\right)_{n} \mathrm{X}$, where $\mathrm{n}=1-4$ and $\mathrm{X}$ is a polar group. The substituents at R3, R4 and R5 are predominantly polar or positively charged. R6, R7 and R8 contained mostly small hydrophobic substituents. The receptor was processed using the Protein Preparation Wizard, which employs a restrained, partial energy minimization (35). The disulfide bond between SrtA and the inhibitor, and the side chain atoms of Cys ${ }^{187}$ were removed to prevent steric clashes during docking. Grids were generated by Glide with the grid box set around the inhibitor using default settings. The inhibitor was excluded in the grid calculations. A docking restraint was set up such that the position of the 5-mercapto sulfur atom of the inhibitor derivatives was restricted within $1 \AA$ of the inhibitor sulfur atom in the NMR structure. Docking was done with Glide using XP settings (36-38). Initially, a total of 623 ligands were docked onto the NMR structure of the protein in the SrtA:inhibitor complex (the coordinates of $\mathbf{2}$-salt were removed prior to docking). After the first round of docking, the best substituents at each site were selected based on two criteria: 1) their docking score was at least one standard deviation above the mean docking score of the control compound 4-ethoxy-5-mercapto-2- 
phenylpyridazin-3(2H)-one, and 2) the substituent made a specific interaction with the protein (e.g. a hydrogen bond). This reduced the number of substituents at R2, R3, R4, R5, R6, R7, and R8 to 19, 8, 12, 7, 4, 1 (hydrogen), and 4, respectively. CombiGlide was then used to generate 2,688 compounds with all possible combinations of substituents at R3, R4, R5, R6 and R7, with R2 restricted to ethoxy and R8 restricted to hydrogen. These 2,688 compounds were docked to the NMR structure and the best substituents were selected as described above. This reduced the number of substituents at R2, R3, R4, R5, R6, R7, and R8 to $19,3,3,1$ (hydrogen), 4, 1 (hydrogen), and 4, respectively. CombiGlide was then used again to generate 2,736 compounds with all possible combinations of substituents, and the compounds were again docked to the NMR structure of the protein. Using the same selection criteria, 12, 3, 3, 1 (hydrogen), 4, 1 (hydrogen), and 1 (hydrogen) substituents were chosen at R2, R3, R4, R5, R6, R7 and R8, respectively. Finally, CombiGlide was then used to generate 432 compounds that contained all possible combinations of the substituents.

The top 432 molecules were docked to the enzyme using a procedure that accounts for SrtA mobility. In this procedure protein motion was first simulated using molecular dynamics (MD) calculations. Bond, angle, and torsion parameters for the inhibitor were derived from the Generalized Amber Force Field (GAFF), using the Antechamber program in Amber (39, 40). Atomic partial charges were derived from RESP (41) fitting of Gaussian09 (42) calculated electrostatic potentials at the Hartree-Fock/6-31G* level. The procedures of MD simulations and clustering were the same as those described in (43). Briefly, a 100-ns conventional MD simulation was performed on the SrtA:inhibitor complex using the AMBER99SB-ILDN force field with the simulation package NAMD $(44,45)$. Eight hundred frames at regularly spaced intervals were extracted from the last $80 \mathrm{~ns}$ of the MD simulation. These frames were aligned by the protein $\mathrm{Ca}$ atoms in the active site and clustered by root mean square deviation (RMSD) conformational clustering using the GROMOS algorithm as implemented in GROMACS 4.5 (46). With an RMSD cutoff of 1.40 $\AA$ A, 25 clusters were obtained, and the centroid member of each cluster was selected to represent each cluster. Subsequently, the top 432 compounds were docked to each of the 25 representative centroid structures, as well as the NMR structure. Procedures used for receptor preparation, grid generation and docking are the same as those described above. To evaluate the docking results, compounds were ranked by the best docking score they obtained from any of the docking calculations to the NMR or 25 centroid conformers. The top 43 compounds were selected as candidates for synthesis and experimental testing.

\section{FRET-based enzymatic assay}

The ability of the compounds to inhibit the activity of SrtA was determined using an established Förster resonance energy transfer (FRET) assay $(19,20)$. The catalytic domains of SrtA (residues $\mathrm{Gln}^{60}$-Lys $^{206}$ ) and Ba-SrtA (residues Asp ${ }^{57}$-Lys ${ }^{210}$ ) were prepared as described previously $(20,47)$. Briefly, in the $\mathrm{IC}_{50}$ assay, $20 \mu \mathrm{L}$ of Sa-SrtA (final assay concentration of $1 \mu \mathrm{M}$ in FRET buffer: $20 \mathrm{mM}$ HEPES, $5 \mathrm{mM} \mathrm{CaCl}_{2}, 0.05 \% \mathrm{v} / \mathrm{v}$ Tween-20, $\mathrm{pH} 7.5$ ) was incubated with $1 \mu \mathrm{L}$ of test compound solution (dissolved in 100\% DMSO, final assay concentration of $0.08-400 \mu \mathrm{M}$ ) for $1 \mathrm{~h}$ at room temperature. Subsequently, $30 \mu \mathrm{L}$ of substrate solution, which consists of the self-quenched fluorogenic peptide Abz-LPETGDap(Dnp)-NH 2 (32 $\mu \mathrm{M}$ final assay concentration) (Peptide 2.0 Inc., Chantilly, VA, USA) 
dissolved in FRET buffer, was added to the mixture. Fluorescence was read immediately using an Infinite ${ }^{\circ}$ M1000 PRO (Tecan US Inc., Morrisville, NC, USA) plate reader with the excitation and emission wavelengths set at 335 and $420 \mathrm{~nm}$, respectively. The $\mathrm{IC}_{50}$ assay used to test Ba-SrtA inhibition was similar with the following adjustments: final assay concentration of Ba-SrtA was $10 \mu \mathrm{M}$, final assay concentration of substrate was $100 \mu \mathrm{M}$, and the FRET buffer was composed of $20 \mathrm{mM}$ HEPES, $0.05 \% \mathrm{v} / \mathrm{v}$ Tween-20, pH 7.5. $\mathrm{IC}_{50}$ values were calculated by fitting three independent sets of data to equation 1 using SigmaPlot 6.0 (SPSS, Inc., Chicago, IL, USA):

$$
\frac{v_{i}}{v_{0}}=\frac{1}{1+\left(\frac{[\mathrm{I}]}{\mathrm{IC}_{50}}\right)^{h}}
$$

where $v_{i}$ and $v_{0}$ are initial velocity of the reaction in the presence and absence of inhibitor at concentration [I], respectively. The term $h$ is Hill's coefficient (48).

For inhibitors that displayed $\mathrm{IC}_{50}$ values lower than half of the enzyme concentration used, data were fit to Morrison's quadratic equation (equation 2) to calculate the apparent dissociation constant $\left(K_{i}^{a p p}\right)$ :

$$
\frac{v_{i}}{v_{0}}=1-\frac{\left([E]_{T}+[I]+K_{i}^{a p p}\right)-\sqrt{\left([E]_{T}+[I]+K_{i}^{a p p}\right)^{2}-4[E]_{T}[I]}}{2[E]_{T}},
$$

where $v_{i}$ and $v_{0}$ are initial velocity of the reaction in the presence and absence of inhibitor at concentration [I], respectively. [E] $]_{\mathrm{T}}$ is the total active enzyme concentration, and $K_{i}^{a p p}$ is the apparent dissociation constant for the enzyme-inhibitor complex (48).

To determine the rate of inhibition of SrtA by compound 2-17, 2-54 and 2-62, the FRET assay we used for $\mathrm{IC}_{50}$ or $K_{i}^{a p p}$ determination was modified such that the Abz-LPETGDap(Dnp)- $\mathrm{NH}_{2}$ substrate and inhibitor of various concentrations (final concentration 2.5 $14 \mu \mathrm{M})$ were added to the enzyme at the same time. Fluorescence was read every $8 \mathrm{~s}$ for a total of $30 \mathrm{~min}$. The reaction progress curve was fit to equation 3 to determine $k_{\mathrm{obs}}$, the rate constant for conversion from the initial velocity phase to full inhibition:

$$
P=\frac{v_{i}}{k_{\text {obs }}}\left[1-\exp \left(-k_{\text {obs }} t\right)\right]
$$

where $\mathrm{P}$ is the baseline-corrected fluorescence value, $v_{i}$ is the initial velocity, and $\mathrm{t}$ is time (48). These $k_{\text {obs }}$ measurements were then plotted against inhibitor concentration and fit to equation 4 to obtain $k_{\text {inact }}$ and $K_{\mathrm{I}}$ values. 


$$
k_{o b s}=\frac{k_{\text {inact }}[I]}{K_{I}+[I]},
$$

where $k_{\text {obs }}$ is the observed rate constant of inhibition at inhibitor concentration [I], $k_{\text {inact }}$ is the maximum rate of inhibition given an infinite concentration of inhibitor, and $K_{\mathrm{I}}$ is the concentration of inhibitor that yields a half-maximum rate of inhibition (48).

\section{Cell wall surface Protein A display assay}

Protocol for the cell-based assay was adapted from Zhang et. al. (49). Overnight cultures of $S$. aureus were diluted 1:100 into tryptic soy broth (TSB) with or without inhibitor supplement and grown at $37^{\circ} \mathrm{C}$ with rotation to an $\mathrm{A}_{600}$ of 0.5. Molecules 2-62, and 2-17 inhibited bacterial growth at $100 \mu \mathrm{M}$, and therefore these cultures were only diluted back 1:10 into TSB; data is normalized to account for background. $600 \mu \mathrm{L}$ aliquots were removed, and $S$. aureus were sedimented by centrifugation $(12,000 \times \mathrm{g}$ for $5 \mathrm{~min})$. Bacteria were suspended in $600 \mu \mathrm{L}$ PBS, $2 \mu \mathrm{L}$ FITC-labeled IgG (Sigma, St. Louis, MO, USA) was added, and samples were incubated at room temperature for $1 \mathrm{~h}$. The cells were again sedimented by centrifugation $(12,000 \times \mathrm{g}$ for $5 \mathrm{~min})$ and washed twice with PBS. Bacterial fluorescence intensity was monitored using the Flex Station (Molecular Devices, Sunnyvale, CA, USA) at 495-nm excitation and 520-nm emission. Sample aliquots were spread on TSB agar plates, and $\mathrm{CFU}$ were determined to derive relative fluorescence units per $\mathrm{A}_{600}$ unit. Triplicate measurements were taken for each trial, and mean and standard deviation were calculated.

\section{Cytotoxicity test}

The cytotoxicity $\mathrm{CC}_{50}$ of each compound was determined using Promega CellTiter-Glo Luminescent Cell Viability Assay kit (Promega Corp., Madison, WI, USA) by measuring the total ATP levels to quantify the number of metabolically active cells upon drug treatment as described in (50). Briefly, the compounds were diluted in 384 plates $(20 \mu 1 /$ well) in triplicate by a 10-point titration ( $195 \mathrm{nM}$ to $100 \mu \mathrm{M})$ followed by the addition of $30 \mu \mathrm{HeLa}$ cells (2500 cells/well). The plates were incubated at $37^{\circ} \mathrm{C}$. Three days later, $50 \mu \mathrm{l}$ of CellTiter-Glo reagent was added to each well followed by a $2 \mathrm{~min}$ shaking and a $10 \mathrm{~min}$ incubation to lyse the cells. The relative luminescent intensity units (RLU) of each well was measured using an Infinite ${ }^{\circledR}$ M1000 PRO (Tecan US Inc., Morrisville, NC, USA) with its green filter and $1 \mathrm{~s}$ integration time. $\mathrm{CC}_{50}$ values were calculated by fitting the three independent sets of data to equation 1 using SigmaPlot 6.0 (SPSS, Inc., Chicago, IL, USA), by replacing $\mathrm{IC}_{50}$ with $\mathrm{CC}_{50}$.

\section{Results}

\section{NMR structure of SrtA bound to a soluble pyridazinone analogue}

Using high throughput screening methods we previously discovered that pyridazinone-based molecules effectively inhibit SrtA (19). One of the most potent molecules that was discovered was 4-ethoxy-5-mercapto-2-phenylpyridazin-3(2H)-one (hereafter referred as 
compound 2-10) (Fig. 1A), which inhibits SrtA's in vitro activity with an $\mathrm{IC}_{50}$ of $13 \pm 1 \mu \mathrm{M}$. However, 2-10 and other pyridazinone-based molecules produced from the screen are poorly soluble in aqueous solvent, and thus could not be optimized using structure-based methods. To overcome this problem, we synthesized a sodium thiolate analogue of 2-10, 5-ethoxy-6oxo-1-phenyl-1,6-dihydropyridazine-4-thiolate (2-salt) (Fig. 1B). 2-salt exhibits substantially improved solubility (up to $75 \mathrm{mM}$ in aqueous buffer) and inhibits the enzyme with an $\mathrm{IC}_{50}$ of $35 \pm 7.4 \mu \mathrm{M}$. Importantly, it's increased solubility enables the productive formation of a SrtA:2-salt complex in which the inhibitor is bound to the catalytic domain of sortase (residues $\mathrm{Gln}^{60}$-Lys ${ }^{206}$ of SrtA). The NMR spectra of the complex are well resolved and differ substantially from apo-form of SrtA (Fig. 1C). Triple resonance methods were used to assign the protein's ${ }^{1} \mathrm{H},{ }^{13} \mathrm{C}$ and ${ }^{15} \mathrm{~N}$ resonances in the SrtA:2-salt complex. A comparison with the previously published chemical shift assignments of apo-SrtA reveals large inhibitor-dependent chemical shift changes in atoms that are located within the enzyme's active site, and only small shift changes for atoms located elsewhere in the protein (Fig. 1C). A comparison of the NOESY spectra of the complex and apo-SrtA reveals that residues located distal to the active site exhibit similar NOE cross peak patterns. We conclude from this data that the inhibitor binds to the enzyme's active site where it causes only localized structural changes.

NMR spectroscopy was used to define the structure of the SrtA:2-salt complex. Nearcomplete chemical shift assignments were obtained for both the protein and bound inhibitor. Because the inhibitor causes only localized changes in the structure of the enzyme, we employed a hybrid approach to determine the structure of the complex. NMR data were used to define the molecular basis of inhibitor binding in the SrtA:2-salt complex (see Methods). A total of 229 experimental restraints define the structure of the enzyme's active site, including: 156 intramolecular protein-protein distance, 20 intermolecular inhibitor-protein NOE distance, $43 \phi$ and $\psi$ dihedral angle, and $10{ }^{3} \mathrm{~J}_{\mathrm{HN}-\mathrm{Ha}}$ coupling constant restraints. An ensemble containing 20 conformers representing the structure of the complex exhibit good covalent geometries and have no NOE, dihedral angle, or scalar coupling violations greater than $0.5 \AA$, $5^{\circ}$, or $2 \mathrm{~Hz}$, respectively. Enzyme interactions with the inhibitor are well defined by the NMR data, as the coordinates of the backbone and heavy atoms within the active site, as well as the inhibitor molecule have a root mean square deviation (RMSD) to the mean structure of $0.20 \pm 0.07$ and $0.69 \pm 0.06 \AA$, respectively. Complete structure and restraint statistics are presented in Table 1.

The structure of the complex reveals that the pyridazinone inhibitor binds to a groove adjacent to the enzyme's active site (Fig. 2A-D). The base of the group was formed by residues in strands $\beta 4$ and $\beta 7$, while residues located in the $\beta 2 / \mathrm{H} 1, \beta 3 / \beta 4, \beta 6 / \beta 7$ and $\beta 7 / \beta 8$ loops form the sides of the groove and partially shield the inhibitor from solvent. Numerous hydrophobic contacts are made to the 2-phenyl group of the inhibitor from the side chains of $\mathrm{Val}^{166}$, Val ${ }^{168}$, and Leu ${ }^{169}$ within the $\beta 6 / \beta 7$ loop, $\mathrm{Val}^{193}$ within the $\beta 7 / \beta 8$ loop, and $\mathrm{Ile}^{182}$ on strand $\beta 6$ (Fig. 2C). These interactions are supported by intermolecular NOEs between the $\mathrm{Val}^{166} \mathrm{Ha}, \mathrm{Val}^{166} \mathrm{H} \gamma, \mathrm{Val}^{168} \mathrm{H} \gamma$, Leu ${ }^{169} \mathrm{H} \delta$, and $\mathrm{Val}^{193} \mathrm{H} \gamma$ protons of the enzyme, and the $\mathrm{H} \varepsilon$ and $\mathrm{H} \delta$ protons of the inhibitor (Fig. 1D). Interestingly, an NOE was identified between the $\mathrm{H} \delta$ proton of Ile ${ }^{182}$ and to the H$\delta$ proton of the inhibitor, whereas no NOEs were 
observed to the inhibitor's $\mathrm{He}$ proton. This is consistent with the structure complex, as the 2phenyl group is only partially buried within a hydrophobic pocket; it is sandwiched between the side chains of $\mathrm{Val}^{193}$ on the $\beta 7 / \beta 8$ loop and Leu ${ }^{169}$ on the $\beta 6 / \beta 7$ loop. The positioning of the central pyridazinone ring of the inhibitor is also well-defined by the NMR data as NOEs between its $\mathrm{H} \gamma$ atom and the $\mathrm{Thr}^{183} \mathrm{Ha}$ (strand $\beta 7$ ), Cys ${ }^{184} \mathrm{Ha}$ (strand $\beta 7$ ), $\operatorname{Trp}^{194} \mathrm{H} \beta$ ( $\beta 7 / \beta 8$ loop), Ile ${ }^{182} \mathrm{H} \gamma 1$ (strand $\beta 7$ ), $\mathrm{Ile}^{182} \mathrm{H \delta}$ (strand $\beta 7$ ), and $\mathrm{Val}^{193} \mathrm{H} \gamma(\beta 7 / \beta 8$ loop) protons are observed (Fig. 1D). This orients the central ring such that the carbonyl group is directed towards the solvent, while the $\mathrm{H} \gamma$ atom faces strand $\beta 7$. The indole ring of $\operatorname{Trp}^{194}$ on the $\beta 7 / \beta 8$ loop closes the inhibitor recognition groove, shielding the disulfide bond between the inhibitor and $\mathrm{Cys}^{184}$ from the solvent. This is evidenced by NOEs between the

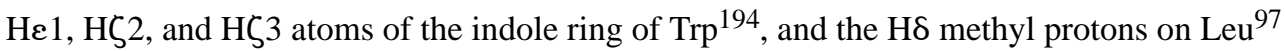
within helix H1. This demonstrates the $\beta 7 / \beta 8$ loop of the $\mathbf{2}$-salt bound enzyme is positioned in a manner more similar to the apo-SrtA enzyme with the loop pressed against H1, and differs from the substrate bound form of the enzyme, as these elements are separated by $\sim 13$ $\AA$ (see Discussion) $(20,32)$. The precise location of 4-ethoxy moiety is not well defined by the NMR data, as only a single NOE defines its positioning (between $\operatorname{Trp}^{194} \mathrm{H} \delta 1$ and Ha methyl of the inhibitor). However, in nearly all conformers within the ensemble it is positioned within a narrow groove formed between the $\beta 7 / \beta 8$ and $\beta 2 / \mathrm{H} 1$ loops.

In the structure of the complex, the sulfhydryl group of the active site cysteine residue $\left(\mathrm{Cys}^{184}\right)$ is positioned adjacent to the 5-mercapto group of the inhibitor, suggesting that they may be joined via a disulfide bond. Although the mechanism of inhibition by the pyridazinone-based compounds is not known, the most potent pyridazinone molecules also contain 5-mercapto group consistent with them also covalently modifying the enzyme. To determine the mechanism of inhibition, we incubated 10-fold molar excess of 2-salt with SrtA, unbound 2-salt was then removed by dialysis and the 2-salt:SrtA complex was digested with trypsin. The digestion products were then analyzed using LC-MS before and after adding DTT. In the absence of DTT, a peak eluting at $6.86 \mathrm{~min}$ has a mass-to-charge ratio consistent with it being a disulfide-linked QLTLITCDDYNEK:2-salt peptide, compatible with cysteine modification (Fig. 3A,B). Moreover, when DTT is added this complex disappears and a new peak corresponding to the unmodified QLTLITCDDYNEK elutes at 5.09 min (Fig. 3A,C). Similar LC-MS results were obtained when the parent compound 2-10 was reacted with the enzyme (data not shown). These data indicate pyridazinone-containing inhibitors such as 2-salt and 2-10 inactivate SrtA by forming disulfide bond to Cys ${ }^{184}$.

\section{Structure-based inhibitor optimization}

Using the atomic coordinates of the SrtA:2-salt complex computational approaches were used to quantitatively predict inhibitor modifications that could increase binding affinity and selectivity. In this procedure, analogues of the 2-10 pyridazinone compound were constructed in silico using the program CombiGlide and their binding poses and energies evaluated using the molecular docking program Glide. Briefly, the 5-mercapto-2phenylpyridazin-3(2H)-one moiety of $\mathbf{2 - 1 0}$ was defined as the core in CombiGlide, and substituents derived from the program's fragment library were then systematically added to eight sites (Fig. 4, sites named R2 to R8). The substituents were chosen based on their 
degree of solvent exposure, and for buried substituents, the accessible volume available to them within the enzyme's subsite. In particular, sites on the small molecule that are solvent exposed (R2-R5) were modeled with predominantly polar groups of various sizes, while sites located on the protein-facing side of the molecule (R6-R8) were modeled to contain mostly small hydrophobic groups (see Materials and Methods). Initially, 6,047 analogues of 2-10 were computationally evaluated, which included 623 containing a single alteration, and 5,424 analogues that contained two alterations at sites R2 to R8 (Fig. 4). Each analogue was then docked to the NMR structure and ranked based on their docking score. Analogues containing single site modifications at R2, R3, R4 and R6 exhibited the largest improvements in binding $(12,3,3$, and 4 analogues containing unique substituents at these sites resulted in improved binding, respectively). Next, to discover small molecules with even greater binding affinity, docking experiments were performed for 432 analogues that each contained four alterations. The small molecules contained substituent changes at their R2, R3, R4 and R6 sites, which were chosen based on the aforementioned single site docking studies. During this phase of the work, the docking calculations made use of the relaxed complex scheme approach to account for protein flexibility in the enzymes active site as previously described $(43,51,52)$. After completing the computational docking experiment, a total of 43 compounds were analyzed further, as their docking scores ranked in top $10 \%$ of the 432 analogs that were tested. These "top" molecules had docking scores ranging from -8.9 to -10.0 , which is a significant improvement as compared to the $\mathbf{2 - 1 0}$ lead molecule (-4.08). An inspection of their chemical structures reveals that modification at sites R2, R4 and R6 are most beneficial $\left(\mathrm{R} 2,\left(-\mathrm{O}\left(\mathrm{CH}_{2}\right)_{2} \mathrm{OH},-\mathrm{O}\left(\mathrm{CH}_{2}\right)_{3} \mathrm{OH},-\mathrm{OCH}_{2} \mathrm{COOH}\right.\right.$, $-\mathrm{O}\left(\mathrm{CH}_{2}\right)_{2} \mathrm{COOH},-\mathrm{OCH}_{2} \mathrm{CONH}_{2}$, and $\left.-\mathrm{O}\left(\mathrm{CH}_{2}\right)_{2} \mathrm{CONH}_{2}\right) ; \mathrm{R} 4,-\mathrm{C}(=\mathrm{NH}) \mathrm{NH}_{2}$ (hereafter referred as amidine); $\mathrm{R} 6,\left(-\mathrm{CH}_{3},-\mathrm{F}\right.$, and $\left.-\mathrm{OCH}_{3}\right)$.

Several promising pyridazinone analogues based on the docking analysis were synthesized and their inhibitory activity determined experimentally using a FRET-based assay (Table 2). Representative dose-response curves are shown in Fig. S1. Analogues were chosen for further study based on their synthetic feasibility and in order to maximize the chemical diversity of the molecular scaffolds that were tested. Initially, ten analogues of 2-10 were synthesized that contained single site changes (2-51 to 2-60). Since 2-10 oxidizes readily to form a more potent and stable symmetric disulfide dimer (2-17), all of the analogs were tested in their oxidized form and their potency compared to 2-17. Improvements of 2- to 10fold were obtained by making single site alterations. In particular, non-polar modifications at site R6 are beneficial, presumably increasing van der Waals contacts to residues in the $\beta 6 / \beta 7$ loop of the enzyme that form a hydrophobic pocket (Fig. 2C,D). In agreement with the structure, the R4 site on the opposite side of the benzene ring is also a good site for the addition of a polar amidine group, which presumably enables contacts to Glu ${ }^{105}$ located in the $\beta 3 / \beta 4$ loop. Altering the length and polar character of the substituent attached to the R2 site is also advantageous, presumably because it enables the formation of additional hydrogen bonds to the residues located within the $\beta 7 / \beta 8$ loop (e.g. Tyr ${ }^{187}$, Gly ${ }^{192}$ and $\operatorname{Trp}^{194}$ ). Two analogs, 2-61 and 2-62, were synthesized that simultaneously alter substituents at sites R2 and R6. Compound 2-61 combines favorable $\mathrm{O}\left(\mathrm{CH}_{2}\right)_{2} \mathrm{OH}(\mathrm{R} 2)$ and $\mathrm{F}(\mathrm{R} 6)$ modifications, but surprisingly did not show a substantial improvement in activity. In contrast, significant improvements in activity are observed in compound 2-62 that combines 
$\mathrm{O}\left(\mathrm{CH}_{2}\right)_{3} \mathrm{OH}(\mathrm{R} 2)$ and $\mathrm{F}(\mathrm{R} 6)$ alterations $\left(\mathrm{IC}_{50}\right.$ value of $\left.0.02 \pm 0.01 \mu \mathrm{M}\right)$. A more complete description of the structure activity relationship analysis is provided in the discussion section.

In order to determine if the compounds had broad spectrum activity against sortase enzymes, we tested their ability to inhibit the Bacillus anthracis class A sortase enzyme (Ba-SrtA), which similar to $S$. aureus SrtA (Sa-SrtA) anchors proteins to the cell wall that contain an LPXTG sorting signal. In general, all of the analogues had good inhibitory activity against Ba-SrtA, with $\mathrm{IC}_{50}$ values in the low micromolar to high nanomolar range. However, distinct species specific trends in inhibitory activity are apparent. For example, the most effective SrtA inhibitor (2-62) is 20-fold less active against Ba-SrtA $\left(\mathrm{IC}_{50}=0.45 \pm 0.29 \mu \mathrm{M}\right.$ against Ba-SrtA as compared to $\mathrm{IC}_{50}=0.02 \pm 0.01 \mu \mathrm{M}$ against Sa-SrtA), whereas the best Ba-SrtA inhibitor compound 2-53 containing a methoxy group at the R3 site is 4-fold more active against Ba-SrtA as compared to SrtA (Ba-SrtA $\mathrm{IC}_{50}=0.15 \pm 0.05 \mu \mathrm{M}$ versus Sa-SrtA IC 50 $=0.62 \pm 0.18 \mu \mathrm{M})$. These subtle differences in potency likely arise from structural differences between the active sites of each enzyme, but nevertheless indicate that pyridazinone-based inhibitors are capable of inhibiting Class A type sortases that recognize LPXTG sorting signals.

\section{Inhibitor inactivation kinetics and effect on protein display in intact cells}

For the parent compound (2-17), positively charged compound (2-54) and the most potent compound based on its $\mathrm{IC}_{50}$ value (2-62), we determined the rate at which they inactivate SrtA. The substrate and inhibitor were simultaneously added to SrtA and the observed rate constant of inhibition $\left(k_{\text {obs }}\right.$ ) was determined by fitting the progress curve to eqn. 3 (see Materials and Methods). Measurement of $k_{\mathrm{obs}}$ at different inhibitor concentrations yields $k_{\text {inact }}$, the rate constant describing inhibitor covalent modification of the enzyme, and $K_{\mathrm{I}}$, which carries the same intrinsic kinetic significance as $K_{\mathrm{m}}$ and approximates the dissociation constant of the non-covalent E-I complex. The overall inactivation efficiency is a function of covalent modification rate and binding affinity $\left(k_{\text {inact }} / K_{\mathrm{I}}\right)$. Fig. $5 \mathrm{~A}$ shows a representative plot of $k_{\mathrm{obs}}$ measured as a function of inhibitor (2-62) concentration, which yields $k_{\text {inact }}$ of $0.034 \pm 0.007 \mathrm{~s}^{-1}, K_{\mathrm{I}}$ of $12 \pm 5 \mu \mathrm{M}$ and $k_{\text {inact }} / K_{\mathrm{I}}$ of $0.003 \pm 0.001 \mu \mathrm{M}^{-1} \mathrm{~s}^{-1}$. Complete results are summarized in Table 3 . Consistent with the $\mathrm{IC}_{50}$ results, inactivation efficiency is significantly higher for 2-62 $\left(0.003 \pm 0.001 \mu \mathrm{M}^{-1} \mathrm{~s}^{-1}\right)$, as compared to 2-54 $\left(0.0006 \pm 0.0003 \mu \mathrm{M}^{-1} \mathrm{~s}^{-1}\right)$ or $\mathbf{2 - 1 7}\left(0.0008 \pm 0.0005 \mu \mathrm{M}^{-1} \mathrm{~s}^{-1}\right)$.

The ability of the most promising inhibitors to disrupt sortase-mediated protein display in intact $S$. aureus cells was determined. In Newman strain of $S$. aureus, SrtA anchors 19 different surface proteins in the bacterial envelope (53), including SpA, a molecule that binds the Fc $\gamma$ and Fab domains of host immunoglobulins (54). The abundance of Ig binding to $\mathrm{SpA}$ in the bacterial cell wall envelope is therefore dependent upon the activity of $S$. aureus SrtA, enabling the effects if the pyridazinone molecules on SpA display to be measured. In the assay, cells are cultured with varying amounts of inhibitor and the amount of displayed SpA then quantified by adding fluorescein isothiocyanate (FITC)-labeled IgG. As expected, a $\Delta s r t A$ control strain displays significantly less SpA on its surface; protein display is reduced by $\sim 80 \%$ in $\Delta s r t A$ as compared to WT $S$. aureus (Fig. 5B). The addition 
of compounds 2-17, 2-54 or 2-62 to WT $S$. aureus cultures caused statistically significant reductions in SpA display. The largest effects are observed when 2-54 is added, which shows a dose-dependent decrease in SpA display. When $100 \mu \mathrm{M}$ is present in the cell culture, display levels are reduced to $35 \%$ of WT, approaching levels observed for the $\Delta s r t A$ strain.

To gain insight into the therapeutic window at which the pyridazinone inhibitors could be dosed to treat $S$. aureus infections their cytotoxicity against human cells was determined. In general, all of the compounds tested exhibit minimal cytotoxicity against human HeLa cells, as they have $\mathrm{CC}_{50}$ values in excess of $100 \mu \mathrm{M}$ (Table 2). Notably, the most potent inhibitor, 2-62, has a $\mathrm{CC}_{50}$ to $\mathrm{IC}_{50}$ ratio as large as 5,000. Interestingly, an analysis of the data reveals that the $\mathrm{CC}_{50}$ values of the compounds are inversely correlated to their predicted octanolwater partition coefficients (cLogP), with molecules that have smaller (more negative) cLogP values exhibiting some of the highest $\mathrm{CC}_{50}$ values. This is not surprising, since a more negative $\operatorname{cLog} P$ value indicates that a compound is more soluble in water, and therefore less likely to be capable of crossing the cellular membrane where it could cause off-target effects that lead to cell death. As SrtA is located on the extracellular membrane of $S$. aureus, additional modification of the pyridazinone analogs to increase their solubility in water may further reduce off target cellular toxicity.

\section{Discussion}

The emergence of methicillin resistant $S$. aureus (MRSA) and other multidrug resistant bacterial pathogens has created an urgent need for new antibiotics. The $S$. aureus SrtA enzyme is an attractive drug target as it covalently attaches virulence factors to the microbial surface that have important functions in the infection process, including mediating bacterial adhesion to host tissues, nutrient acquisition and the suppression and evasion of the immune system. SrtA's importance has been validated in animal models of infection that demonstrate that $S$. aureus sit $A^{-}$mutants have attenuated virulence. To date, several synthetic, peptidebased and natural product inhibitors of SrtA have been reported (55), but only a few investigators have been able to rationally optimize these molecules using structural biology approaches. This is because it has been problematic to study the enzyme using x-ray crystallography, since its active site exhibits a high degree of conformational disorder that hinders crystallization. Moreover, the conformational disorder makes accurate computational modeling of enzyme-inhibitor interactions challenging. To overcome these problems, we employed a novel soluble pyridazinone analogue that enabled the NMR structure enzymepyridazinone to be determined. Using the structure of the complex we rationally optimized several pyridazinone-based inhibitors of SrtA, leading to new second-generation molecules that have improved inhibitory activity and lower cellular toxicity.

Our structural studies of the water soluble pyridazinone inhibitor, 2-salt, reveal that it causes a disordered to ordered structural change in the enzyme's active site that is reminiscent of the previously documented changes caused by binding the sorting signal substrate. Our previous NMR studies of SrtA in its apo-state have revealed that it contains a large active site loop that connects strands $\beta 6$ to $\beta 7$ (the $\beta 6 / \beta 7$ loop). In the absence of the substrate, the loop is structurally disordered and undergoes motions on the micro- to millisecond time scale (20). However, upon binding the LPXTG sorting signal, motions in the loop are 
quenched and as a result of a disordered to ordered conformational change that enables it to partially encapsulate the leucine side chain within the bound peptide (32). Interestingly, in the structure of the SrtA:2-salt complex, the inhibitor binds to the same site on SrtA as the LPXTG sorting signal, and similar to the substrate it alters the structure and dynamics of the $\beta 6 / \beta 7$ active site loop (Fig. 6). In the SrtA-sorting signal substrate complex, residues Val ${ }^{166}$ Leu ${ }^{169}$ the $\beta 6 / \beta 7$ loop form a $3_{10}$ helix that facilitates closure over the substrate by forming a non-polar surface that contacts the leucine side chain. In the SrtA:2-salt complex the 2phenyl group in the inhibitor mimics the leucyl side chain, as contacts to it drive loop

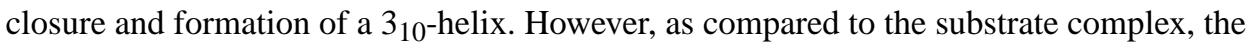

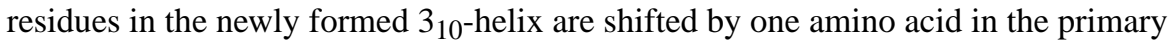
sequence; in the SrtA:2-salt and SrtA-substrate complexes, the newly formed active site helix is comprised of residues Gly ${ }^{167}$-Asp ${ }^{170}$ and residues Val ${ }^{166}$ Leu $^{169}$, respectively. This subtle shift is presumably caused by inhibitor contacts originating from side chains of $\mathrm{Val}^{166}$ and Leu ${ }^{169}$, which in the SrtA:2-salt complex pack against the 2-phenyl group of the inhibitor necessitating that the backbone atoms of $\mathrm{Val}^{166}$ adopt an extended conformation (Fig. 6D). In contrast, in the SrtA:substrate complex, Val ${ }^{166}$ adopts a helical conformation such that its side chain is rotated away from the active site so as to accommodate the leucine side chain of the sorting signal substrate (Fig. 6E).

The pyridazinone inhibitor does not fully mimic the sorting signal substrate as its binding only partially closes the $\beta 6 / \beta 7$ loop and it fails to substantially reposition the $\beta 7 / \beta 8$ loop active site loop. In our previously reported structure of the SrtA-substrate complex, in addition to inducing a $3_{10}$ helix formation within the $\beta 6 / \beta 7$ loop, substrate binding also caused a disordered to ordered transition in residues that immediately preceded the helix in the primary sequence. These changes fully closed the loop, and presumably constructed a catalytically competent active site in which the side chain guanidino group of $\operatorname{Arg}^{197}$ is positioned to stabilize high-energy anionic reaction intermediates. However, in the structure of the inhibitor complex, the active site does not fully form as this region of the $\beta 6 / \beta 7$ loop remains flexible and disordered. This is evidenced by the absence of NMR resonances for residues Thr ${ }^{156}$ - Asp $^{165}$ in the $\beta 6 / \beta 7$ loop in both the apo- and inhibitor bound forms of the enzyme, which are presumably broadened beyond detection because of motions on micro- to milli-second time scale (Fig. 6C). This notion is also substantiated by the absence of signals for residues $\mathrm{Arg}^{197}-\mathrm{Phe}^{200}$ in strand $\beta 8$ in both forms of the enzyme, which are presumably rigid, but broadened beyond detection because their magnetic environments fluctuate as a result of motions in the in proximal $\beta 6 / \beta 7$ loop. A comparison of the substrate- and inhibitor-bound forms of the enzyme also reveals other key differences. Previously we have shown that upon binding the signal analogue the $\beta 7 / \beta 8$ loop in SrtA is displaced by $\sim 13 \AA$. This rearrangement results in the formation of a new groove between the $\beta 7 / \beta 8$ loop and helix H1, which has been hypothesized to form the binding site of the secondary substrate, the Gly 5 cross-bridge of lipid II. Interestingly, binding of the inhibitor displaces the loop by only $\sim 3.5 \AA$, such that the groove is not exposed. This is substantiated by presence of NOEs in the spectra of the inhibitor complex between $\mathrm{H \varepsilon} 1, \mathrm{H} \zeta 2$, and $\mathrm{H} \zeta 3$ of the indole ring of $\operatorname{Trp}^{194}$ located in the $\beta 7 / \beta 8$ loop and H $\delta$ methyl protons on $\mathrm{Leu}^{97}$ within helix H1 (similar NOEs are present in the NMR spectra of the apo-form of the enzyme, but absent in the SrtAsubstrate complex). Thus, although the inhibitor triggers partial closure of the $\beta 6 / \beta 7$ loop 
and the $3_{10}$ helix formation similar to the sorting signal substrate, the changes are less substantial and the conformation and dynamics of residues in the $\beta 6 / \beta 7$ loop nearest the active site, as well as the $\beta 7 / \beta 8$ loop are not significantly affected by inhibitor binding.

The structure of the SrtA:2-salt complex is the first high-resolution structure of a SrtA-type enzyme bound to a small molecule inhibitor. Previously, Zhulenkovs and colleagues solved the NMR structure of $S$. aureus SrtA in complex with a benzisothiazolinone-based inhibitor (16). However, the coordinates of the inhibitor were not well-defined, since only nine intermolecular enzyme-inhibitor NOEs defined its positioning. The authors speculated that both the $\beta 6 / \beta 7$ and $\beta 7 / \beta 8$ loops in the SrtA- benzisothiazolinone complex are partially disordered such that the bound inhibitor can adopt different conformations. This is distinct from pyridazinone-based molecules, since in the SrtA:2-salt complex a portion of the $\beta 6 / \beta 7$ loop near the inhibitor becomes structurally ordered and the $\beta 7 / \beta 8$ loop is structurally ordered and adopts a closed conformation that is similar to its conformation in the apo-form of the protein. Nevertheless, their binding mechanisms share two common features. First, both pyridazinone and benzisothiazolinone inhibitors covalently modify SrtA by forming a disulfide bond with Cys ${ }^{184}$. Second, despite the flexibility of the benzisothiazolinone compound and the partially disordered active site loops, in both inhibitor complexes the sidechains of $\mathrm{Val}^{166}$, $\mathrm{Val}^{168}$ and Leu ${ }^{169}$ in the $\beta 6 / \beta 7$ loop and $\operatorname{Trp}^{194}$ in the $\beta 7 / \beta 8$ loop consistently interact with the bound inhibitor. Structures of the SrtB sortase have also been determined in complex with small molecule inhibitors. To aid development of aryl ( $\beta$ amino)ethyl ketone (AAEK)-based inhibitors, Maresso and colleagues solved the crystal structures of $B$. anthracis SrtB bound to two different AAEK compounds (17). Similar to 2salt and the benzisothiazolinone compound, AAEK covalently modifies the active site cysteine residue (Cys ${ }^{233}$ in SrtB). Interestingly, while AAEK does not make specific contacts with residues within the active site loops, the compound nevertheless induces ordering of the $\beta 7 / \beta 8$ loop and partial disordering of the $\beta 6 / a 5$ loop (SrtB equivalent of the $\beta 6 / \beta 7$ loop). Combined, structural studies of sortase-inhibitor complexes reveal that sortase inhibitors could modulate the active site loops dynamics, and high affinity binding might be achieved by maximizing contacts with the residues within the active site loops.

Rational approaches were employed to identify pyridazinone analogues with improved inhibitory activity. Concurrently, we also sought to increase the aqueous solubility of the inhibitors by adding polar substituents to sites on the inhibitor that based on the structure of the complex are surface exposed. This was done to diminish the capacity of the inhibitor to cross the host-cell membrane where it could cause undesirable off-target effects. As described in the results section, analogues of $\mathbf{2 - 1 0}$ containing a single alteration at site R2 to R6 were computationally evaluated for their ability to bind SrtA using the structure of the complex as a template. This identified substituents at each site that improved binding affinity, which were then tested in combination using docking calculations that employed the relaxed complex scheme approach to account for protein flexibility. Analogues that scored the highest in this analysis were then synthesized and experimentally tested. This led to a total of seven new analogues that have improved inhibitory activity against SrtA.

Modification of the ortho positions of the 2-phenyl ring have a large impact on activity and solubility. Based on the structure of the complex, substituents at these sites either project 
into the solvent (R4), or they contact hydrophobic residues in the active site loop (R6).

Compounds 2-51 to $\mathbf{2 - 5 3}$ selectively modify position R6 on the scaffold, and show improved activity when bulkier fluoro (2-51) or O-methyl groups (2-53) are added, resulting in 9- and 2 -fold improvements in inhibitory activity, respectively. Based on the structure, these modifications fill a hydrophobic pocket on the enzyme that is formed by non-polar side chains originating from the $\beta 6 / \beta 7$ loop ( $\mathrm{Val}^{166}, \mathrm{Val}^{168}$, and $\mathrm{Leu}^{169}$ ), the $\beta 7 / \beta 8$ loop ( $\mathrm{Val}^{193}$ ) and the underlying beta sheet (Ile ${ }^{182}$ in strand $\beta 7$ ). Interestingly, only small improvements are observed for 2-52, presumably because this analogue adds a methyl group at site R6 that may be too small to adequately fill the pocket. In contrast, while a fluoro group is slightly smaller than a methyl group, it is capable of forming multipolar contacts with the protein in addition to hydrophobic interactions (56), which might explain the bigger improvement in activity of 2-51 over 2-52. Similar to site R6, the R4 site is in an ortho position, but it is located on the opposite side of the phenyl ring where it is surface exposed and projected towards an anionic patch on the enzyme that is formed by the side chains of $\mathrm{Glu}^{105}$ and $\mathrm{Asp}^{170}$. The positioning of the $\mathrm{R} 4$ substituent in the structure of the complex is compatible with the inhibitory properties of compound $\mathbf{2 - 5 4}$, which exhibits a $\sim 3$-fold reduction in its $\mathrm{IC}_{50}$ relative to the lead molecule. This is because this analogue contains an amidine substituent at site R4 which can presumably form favorable electrostatic interactions. Notably, the addition of a polar amidine substituent at this site substantially decreases the cLogP score as compared to the parent molecule, suggesting that polar substituent addition to site R4 is a viable strategy for improving inhibitor solubility.

Based on the docking studies a total of 12 analogues containing alterations at site R2 were synthesized and tested experimentally. Based on the structure of the complex R2 substituents rest on a surface exposed groove that is located in between the $\beta 7 / \beta 8$ and $\beta 2 / \mathrm{H} 1$ loops. The addition of hydroxyl bearing 2-hydroxyethoxy (2-55) and 3-hydroxypropoxy (2-56) at site R2 led to 6.8- and 1.8-fold improved activity, respectively. Although the coordinates of the R2 substituent (4-ethoxy) are poorly defined in the structure of SrtA:2-salt, the improved binding of these molecules may result from their ability to form additional hydrogen bonds via their hydroxyl group to residues within the $\beta 2 / \mathrm{H} 1$ or $\beta 7 / \beta 8$ loops. Interestingly, this poorly defined subsite on SrtA does not accommodate large carboxylic acid and amide substituents at site R2, as analogues containing these modifications (2-57 to 2-60) have increased $\mathrm{IC}_{50}$ values. In addition to $S$. aureus $\mathrm{SrtA}$, we also tested each compound's ability to inhibit the activity of the SrtA sortase enzyme from B. anthracis (Ba-SrtA), another important human pathogen that causes anthrax disease (57). SrtA and Ba-SrtA recognize related LPXTG sorting signals and their active sites adopt similar, but non-identical atomic structures (47). As with SrtA, modifications at sites R2 and R6 in the lead molecule led to improved inhibitory activity. The greatest gains in activity occur when the R6 site is modified with a methoxy group (2-53) (8-fold improved activity as compared to the lead molecule). However, distinct trends in the SAR data for SrtA and Ba-SrtA are evident. For example, in contrast to SrtA, modifying the $\mathrm{R} 4$ site with amidine led to an increase in $\mathrm{IC}_{50}$. This is presumably because the bacillus enzyme lacks the aforementioned acidic residues that are presumed to stabilize binding to SrtA. Combined, experimental testing of the computationally optimized analogs of $\mathbf{2 - 1 0}$ substantiates the importance of three sites on the 
pyridazinone scaffold (R2, R4 and R6), which when changed individually improve inhibitory activity against SrtA up to 9 fold.

Modifying two sites on the pyridazinone scaffold yielded the most potent sortase inhibitor, compound 2-62 (2-(3-fluorophenyl)-4-(3-hydroxypropoxy)-5-mercaptopyridazin-3(2H)one). It is 70-times more active than the lead compound ( $\mathrm{IC}_{50}$ of $21 \mathrm{nM}$ ) and contains 3hydroxypropoxy and fluoro groups at sites R2 and R6, respectively. Its activity indicates that modification of sites R2 and R6 can have an additive effect, as individually these alterations are predicted to improve binding 63-fold (6.8-fold for site R2 and 9-fold for site R6). The docking pose of 2-62 suggests that its improved binding can be attributed to an increase in the van der Waals contact surface with the enzyme afforded by addition of a fluoro group at position R6 ( $10 \AA^{2}$ increase in buried surface area) (Fig. 7A,C). In addition, binding may be aided by new favorable interactions to the $\beta 7 / \beta 8$ loop formed from the terminal hydroxyl on the $\mathrm{O}\left(\mathrm{CH}_{2}\right)_{3} \mathrm{OH}$ group, as it is positioned to donate and accept a hydrogen bond from the backbone carbonyl oxygen of Gly ${ }^{192}$ and side chain hydroxyl group of $\mathrm{Tyr}^{187}$, respectively. Moreover, in the 2-62 docking pose the oxygen at the R2 site closest to the pyridazinone ring is positioned to accept a hydrogen bond from the $\varepsilon \mathrm{NH}$ group in the indole ring of Trp ${ }^{194}$. Interestingly, a close analogue of 2-62 that contains $\mathrm{O}\left(\mathrm{CH}_{2}\right)_{2} \mathrm{OH}$ at position $\mathrm{R} 2$ instead of $\mathrm{O}\left(\mathrm{CH}_{2}\right)_{3} \mathrm{OH}$ (compound 2-61) is 20 times less potent than 2-62. Insight into its reduced potency is provided by its docking pose, which reveals that its shorter R2 side chain does not favorably hydrogen bond with $\mathrm{Tyr}^{187}$ and Gly ${ }^{192}$, but instead forms a single hydrogen bond to the backbone oxygen of $\mathrm{Ala}^{92}$ located in the $\beta 2 / \mathrm{H} 1$ loop (Fig. 7B,D).

Because the pyridazinone molecules covalently inactivate sortase, the $\mathrm{IC}_{50}$ values reported in Table 2 may not accurately define each inhibitor's potency, as this parameter does not reveal the rate at which the small molecules modify and inactivate the enzyme. We therefore measured the $k_{\text {inact }}$ and $K_{\mathrm{I}}$ parameters for 2-17, 2-54 and 2-62, which were also tested using the cellular assay. As reported in Table 3, 2-62 has the highest inactivation efficiency $\left(k_{\text {inact }} / K_{\mathrm{I}}=0.003 \mu \mathrm{M}^{-1} \mathrm{~s}^{-1}\right.$ ), which is consistent with it having the lowest measured $\mathrm{IC}_{50}$ value when it is pre-incubated with the enzyme for one hour (Table 2). Although 2-10 was not tested due to its tendency to oxidize into $2-17$, presumably the $\sim 10$-fold lower $\mathrm{IC}_{50}$ of 2-17 as compared to 2-10 is caused by a difference in their $k_{\text {inact }}$ values. This is because it is expected that 2-17 will have a higher $k_{\text {inact }}$ value as it inactivates sortase's Cys ${ }^{184}$ residue via a thiol-disulfide exchange mechanism, whereas 2-10 inactivates sortase via a slower thiol-thiol oxidation process. Notably, 2-62 inactivates SrtA significantly more efficiently than previously reported diazoketone or chloromethylketone containing peptidomimics, which exhibited $k_{\text {inact }} / K_{\mathrm{I}}$ values of $0.0004 \mu \mathrm{M}^{-1} \mathrm{~s}^{-1}$ and $0.0009 \mu \mathrm{M}^{-1} \mathrm{~s}^{-1}$, respectively (58). This is presumably a result of the higher reactivity of the sulfhydryl group that we have shown modifies the active site cysteine residue (Fig. 3). Importantly, the SrtA inhibitors are capable of reducing protein display in intact $S$. aureus cells, as incubating analogues 2-17, 2-54 and 2-62 with bacterial cell cultures dramatically reduces $\mathrm{SpA}$ reporter protein display (Fig. 5B). Interestingly, although 2-62 is the most potent compound in vitro based on its $\mathrm{k}_{\text {inact }} / K_{\mathrm{I}}$ and $\mathrm{IC}_{50}$ values, 2-54 is nonetheless more effective than 2-17 or 2-62 at inhibiting SpA display in intact cells. This could be due to the positive charge on the amidine group in $\mathbf{2 - 5 4}$, which may better allow it to traverse the cell wall as compared to the less polar $\mathbf{2 - 1 7}$ 
and 2-62 molecules. This idea is consistent with the finding that 2-17 and 2-62 do not exhibit dose-dependent activity in the cellular assay when their concentration is increased from $50 \mu \mathrm{M}$ to $100 \mu \mathrm{M}$. Presumably their higher cLogP values as compared to $\mathbf{2 - 5 4}$ cause them to be less soluble in the growth media used in cellular assay, thereby limiting the effective inhibitor concentration that can be achieved at the cell surface. In general, our inhibitors are less active in the cellular assay as compared to the in vitro assay. It is conceivable that their effective concentration in the cellular assay is reduced because they non-specifically interact with the bacterial cell wall, or because they interact with components that are uniquely present in the growth media (e.g. secreted proteins). In particular, the presence of low molecular weight thiol containing compounds in the media (e.g. glutathione or coenzyme A) could inactivate the inhibitors by covalently modifying their thiol group. Importantly, although complete disruption of display was not achieved at $100 \mu \mathrm{M}$, the in vitro and cellular potencies of our compounds are similar to, or better than, recently described SrtA inhibitors that are efficacious in treating $S$. aureus infections in a mouse model (49).

\section{Conclusions}

In summary, using NMR spectroscopy, mass spectrometry and a designed soluble analogue, we have determine the mechanism through which pyridazinone-based small molecules inhibit the $S$. aureus SrtA enzyme. We demonstrate that these inhibitors partially mimic SrtA's natural substrate by partially inducing a disordered to ordered conformational change in the $\beta 6 / \beta 7$ active site loop. Using computational and synthetic chemistry approaches, several second-generation inhibitors have been produced that have increased inhibitory activity, both in vitro and on the bacterial cell surface. At present, two SrtA inhibitors have now been reported that are efficacious in treating potentially lethal $S$. aureus infections in animal models. These molecules include, (Z)-3-(2,5-dimethoxyphenyl)-2-(4methoxyphenyl) acrylonitrile (DMMA) and 3-(4-pyridinyl)-6-(2- sodiumsulfonatephenyl) [1,2,4]triazolo[3,4-b][1,3,4]thiadiazole (triazolothiadiazole), which were efficacious when dosed at 20 and $40 \mathrm{mg}$ of compound per $\mathrm{kg}$ of animal, respectively $(49,59)$. The 2-62 pyridazinone compound reported in this paper has a lower $\mathrm{IC}_{50}$ than these molecules; 2-62 has an $\mathrm{IC}_{50}=0.02 \mu \mathrm{M}$, versus $\mathrm{IC}_{50}$ values of $9.2 \mu \mathrm{M}$ and $9.3 \mu \mathrm{M}$ for DMMA and triazolothiadiazol, respectively. This suggests that 2-62 and related pyridazinone molecules are potential candidates for further development into anti-infective agents. Such new therapeutics are needed, as many microbial pathogens are increasingly becoming resistant to current antibiotic therapies.

\section{Supplementary Material}

Refer to Web version on PubMed Central for supplementary material.

\section{Acknowledgments}

This research was supported by funding from the National Institutes of Health (AI52217 to R.T.C. and M.E.J.; and GM31749 to J.A.M.). A.H.C. was supported by the UCLA Chemistry Biology Interface Training program (NIH T32GM008496). B.R.A was supported by the UCLA-MBI Whitcome Pre-Doctoral Training Grant. Additional support at UCSD has been provided by the National Science Foundation, the Howard Hughes Medical Institute, the Center for Theoretical Biological Physics, the National Biomedical Computation Resource, and the NSF 
Supercomputer Centers. Mass Spectrometry Instrumentation was made available through the support of Greg Khitrov (University of California, Los Angeles Molecular Instrumentation Center - Mass Spectrometry Facility in the Department of Chemistry). The mass spectrometry data was collected through a project described and supported by Grant Number S10-RR025631 from the National Center for Research Resources.

\section{Abbreviations}

MRSA Methicillin resistant Staphylococcus aureus

SrtA Staphylococcus aureus sortase A

Ba-SrtA Bacillus anthracis sortase A

Ba-SrtB Bacillus anthracis sortase B

AAEK $\quad \operatorname{aryl}(\beta$-amino)ethyl ketone

\section{References}

1. Lowy FD. Staphylococcus aureus infections. N Engl J Med. 1998; 339:520-32. [PubMed: 9709046]

2. Pallin DJ, Egan DJ, Pelletier AJ, Espinola JA, Hooper DC, Camargo CA Jr. Increased US emergency department visits for skin and soft tissue infections, and changes in antibiotic choices, during the emergence of community-associated methicillin-resistant Staphylococcus aureus. Ann Emerg Med. 2008; 51:291-8. [PubMed: 18222564]

3. McCaig LF, McDonald LC, Mandal S, Jernigan DB. Staphylococcus aureus-associated skin and soft tissue infections in ambulatory care. Emerg Infect Dis. 2006; 12:1715-23. [PubMed: 17283622]

4. Welsh KJ, Abbott AN, Lewis EM, Gardiner JM, Kruzel MC, Lewis CT, Mohr JF, Wanger A, Armitige LY. Clinical characteristics, outcomes, and microbiologic features associated with methicillin-resistant Staphylococcus aureus bacteremia in pediatric patients treated with vancomycin. J Clin Microbiol. 2010; 48:894-9. [PubMed: 20089758]

5. Marty FM, Yeh WW, Wennersten CB, Venkataraman L, Albano E, Alyea EP, Gold HS, Baden LR, Pillai SK. Emergence of a clinical daptomycin-resistant Staphylococcus aureus isolate during treatment of methicillin-resistant Staphylococcus aureus bacteremia and osteomyelitis. J Clin Microbiol. 2006; 44:595-7. [PubMed: 16455920]

6. Ikeda-Dantsuji Y, Hanaki H, Sakai F, Tomono K, Takesue Y, Honda J, Nonomiya Y, et al. Linezolidresistant Staphylococcus aureus isolated from 2006 through 2008 at six hospitals in Japan. J Infect Chemother. 2011; 17:45-51. [PubMed: 20607345]

7. Navarre WW, Schneewind O. Surface proteins of gram-positive bacteria and mechanisms of their targeting to the cell wall envelope. Microbiol Mol Biol Rev. 1999; 63:174-229. [PubMed: 10066836]

8. Foster TJ, Geoghegan JA, Ganesh VK, Hook M. Adhesion, invasion and evasion: the many functions of the surface proteins of Staphylococcus aureus. Nat Rev Microbiol. 2014; 12:49-62. [PubMed: 24336184]

9. Mazmanian SK, Liu G, Jensen ER, Lenoy E, Schneewind O. Staphylococcus aureus sortase mutants defective in the display of surface proteins and in the pathogenesis of animal infections. Proc Natl Acad Sci U S A. 2000; 97:5510-5. [PubMed: 10805806]

10. Cascioferro S, Totsika M, Schillaci D. Sortase A: an ideal target for anti-virulence drug development. Microb Pathog. 2014; 77:105-12. [PubMed: 25457798]

11. Spirig T, Weiner EM, Clubb RT. Sortase enzymes in Gram-positive bacteria. Mol Microbiol. 2011; 82:1044-59. [PubMed: 22026821]

12. Mazmanian SK, Liu G, Ton-That H, Schneewind O. Staphylococcus aureus sortase, an enzyme that anchors surface proteins to the cell wall. Science. 1999; 285:760-3. [PubMed: 10427003]

13. Rasko DA, Sperandio V. Anti-virulence strategies to combat bacteria-mediated disease. Nat Rev Drug Discov. 2010; 9:117-28. [PubMed: 20081869] 
14. Escaich S. Antivirulence as a new antibacterial approach for chemotherapy. Curr Opin Chem Biol. 2008; 12:400-8. [PubMed: 18639647]

15. Suree N, Jung ME, Clubb RT. Recent advances towards new anti-infective agents that inhibit cell surface protein anchoring in Staphylococcus aureus and other gram-positive pathogens. Mini Rev Med Chem. 2007; 7:991-1000. [PubMed: 17979801]

16. Zhulenkovs D, Rudevica Z, Jaudzems K, Turks M, Leonchiks A. Discovery and structure-activity relationship studies of irreversible benzisothiazolinone-based inhibitors against Staphylococcus aureus sortase A transpeptidase. Bioorg Med Chem. 2014; 22:5988-6003. [PubMed: 25282649]

17. Maresso AW, Wu R, Kern JW, Zhang R, Janik D, Missiakas DM, Duban ME, Joachimiak A, Schneewind $\mathrm{O}$. Activation of inhibitors by sortase triggers irreversible modification of the active site. J Biol Chem. 2007; 282:23129-39. [PubMed: 17545669]

18. Zhang R, Wu R, Joachimiak G, Mazmanian SK, Missiakas DM, Gornicki P, Schneewind O, Joachimiak A. Structures of sortase B from Staphylococcus aureus and Bacillus anthracis reveal catalytic amino acid triad in the active site. Structure. 2004; 12:1147-56. [PubMed: 15242591]

19. Suree N, Yi SW, Thieu W, Marohn M, Damoiseaux R, Chan A, Jung ME, Clubb RT. Discovery and structure-activity relationship analysis of Staphylococcus aureus sortase A inhibitors. Bioorg Med Chem. 2009; 17:7174-85. [PubMed: 19781950]

20. Ilangovan U, Ton-That H, Iwahara J, Schneewind O, Clubb RT. Structure of sortase, the transpeptidase that anchors proteins to the cell wall of Staphylococcus aureus. Proc Natl Acad Sci U S A. 2001; 98:6056-61. [PubMed: 11371637]

21. Delaglio F, Grzesiek S, Vuister GW, Zhu G, Pfeifer J, Bax A. NMRPipe: a multidimensional spectral processing system based on UNIX pipes. J Biomol NMR. 1995; 6:277-93. [PubMed: 8520220]

22. Garrett DS, Powers R, Gronenborn AM, Clore GM. A common sense approach to peak picking in two-, three-, and four-dimensional spectra using automatic computer analysis of contour diagrams. 1991. J Magn Reson. 1991; 95:214-20.

23. Keller, R. The Computer Aided Resonance Assignment Tutorial. Goldau, Switzerland: CATINA Verlag; 2004.

24. Cavanagh, J., Fairbrother, WJ., Palmer, AG., Skelton, NJ. Protein NMR spectroscopy. 2. San Diego, CA: Elsevier Science and Technology; 2006.

25. Teng, Q. Structural Biology: Practical NMR Applications. New York: Springer Verlag; 2005.

26. Shen Y, Delaglio F, Cornilescu G, Bax A. TALOS+: a hybrid method for predicting protein backbone torsion angles from NMR chemical shifts. J Biomol NMR. 2009; 44:213-23. [PubMed: 19548092]

27. Vuister GW, Bax A. Quantitative J correlation: a new approach for measuring homonuclear threebond J(HNH.alpha.) coupling constants in 15N-enriched proteins. J Am Chem Soc. 1993; 115:7772-7.

28. Herrmann T, Guntert P, Wuthrich K. Protein NMR structure determination with automated NOEidentification in the NOESY spectra using the new software ATNOS. J Biomol NMR. 2002; 24:171-89. [PubMed: 12522306]

29. Herrmann T, Guntert P, Wuthrich K. Protein NMR structure determination with automated NOE assignment using the new software CANDID and the torsion angle dynamics algorithm DYANA. J Mol Biol. 2002; 319:209-27. [PubMed: 12051947]

30. Schwieters CD, Kuszewski JJ, Tjandra N, Clore GM. The Xplor-NIH NMR molecular structure determination package. J Magn Reson. 2003; 160:65-73. [PubMed: 12565051]

31. Zong Y, Bice TW, Ton-That H, Schneewind O, Narayana SV. Crystal structures of Staphylococcus aureus sortase A and its substrate complex. J Biol Chem. 2004; 279:31383-9. [PubMed: 15117963]

32. Suree N, Liew CK, Villareal VA, Thieu W, Fadeev EA, Clemens JJ, Jung ME, Clubb RT. The structure of the Staphylococcus aureus sortase-substrate complex reveals how the universally conserved LPXTG sorting signal is recognized. J Biol Chem. 2009; 284:24465-77. [PubMed: 19592495]

33. Koradi R, Billeter M, Wuthrich K. MOLMOL: a program for display and analysis of macromolecular structures. J Mol Graph. 1996; 14:51-5. 29-32. [PubMed: 8744573] 
34. DeLano, WL. The PyMOL Molecular Graphics System. Palo Alto, CA: DeLano Scientific, LLC; 2006. The PyMOL Molecular Graphics System.

35. Sastry GM, Adzhigirey M, Day T, Annabhimoju R, Sherman W. Protein and ligand preparation: parameters, protocols, and influence on virtual screening enrichments. J Comput Aided Mol Des. 2013; 27:221-34. [PubMed: 23579614]

36. Halgren TA, Murphy RB, Friesner RA, Beard HS, Frye LL, Pollard WT, Banks JL. Glide: a new approach for rapid, accurate docking and scoring. 2. Enrichment factors in database screening. $\mathbf{J}$ Med Chem. 2004; 47:1750-9. [PubMed: 15027866]

37. Friesner RA, Banks JL, Murphy RB, Halgren TA, Klicic JJ, Mainz DT, Repasky MP, Knoll EH, Shelley M, Perry JK, Shaw DE, Francis P, Shenkin PS. Glide: a new approach for rapid, accurate docking and scoring. 1. Method and assessment of docking accuracy. J Med Chem. 2004; 47:1739-49. [PubMed: 15027865]

38. Friesner RA, Murphy RB, Repasky MP, Frye LL, Greenwood JR, Halgren TA, Sanschagrin PC, Mainz DT. Extra precision glide: docking and scoring incorporating a model of hydrophobic enclosure for protein-ligand complexes. J Med Chem. 2006; 49:6177-96. [PubMed: 17034125]

39. Wang J, Wang W, Kollman PA, Case DA. Automatic atom type and bond type perception in molecular mechanical calculations. J Mol Graph Model. 2006; 25:247-60. [PubMed: 16458552]

40. Wang J, Wolf RM, Caldwell JW, Kollman PA, Case DA. Development and testing of a general amber force field. J Comput Chem. 2004; 25:1157-74. [PubMed: 15116359]

41. Cornell WD, Cieplak P, Bayly CI, Kollman PA. Application of Resp Charges to Calculate Conformational Energies, Hydrogen-Bond Energies, and Free-Energies of Solvation. Journal of the American Chemical Society. 1993; 115:9620-31.

42. Frisch, MJ., Trucks, GW., Schlegel, HB., Scuseria, GE., Robb, MA., Cheeseman, JR., Scalmani, G., et al. Gaussian 09. Wallingford, CT, USA: Gaussian, Inc; 2009. Gaussian 09.

43. Chan AH, Wereszczynski J, Amer BR, Yi SW, Jung ME, McCammon JA, Clubb RT. Discovery of Staphylococcus aureus sortase A inhibitors using virtual screening and the relaxed complex scheme. Chem Biol Drug Des. 2013; 82:418-28. [PubMed: 23701677]

44. Lindorff-Larsen K, Piana S, Palmo K, Maragakis P, Klepeis JL, Dror RO, Shaw DE. Improved side-chain torsion potentials for the Amber ff99SB protein force field. Proteins. 2010; 78:1950-8. [PubMed: 20408171]

45. Phillips JC, Braun R, Wang W, Gumbart J, Tajkhorshid E, Villa E, Chipot C, Skeel RD, Kale L, Schulten K. Scalable molecular dynamics with NAMD. J Comput Chem. 2005; 26:1781-802. [PubMed: 16222654]

46. Pronk S, Pall S, Schulz R, Larsson P, Bjelkmar P, Apostolov R, Shirts MR, Smith JC, Kasson PM, van der Spoel D, Hess B, Lindahl E. GROMACS 4.5: A high-throughput and highly parallel open source molecular simulation toolkit. Bioinformatics. 2013; 29:845-54. [PubMed: 23407358]

47. Weiner EM, Robson S, Marohn M, Clubb RT. The Sortase A enzyme that attaches proteins to the cell wall of Bacillus anthracis contains an unusual active site architecture. J Biol Chem. 2010; 285:23433-43. [PubMed: 20489200]

48. Copeland, RA. Evaluation of Enzyme Inhibitors in Drug Discoveries: A Guide for Medicinal Chemists and Pharmacologists. New Jersey: John Wiley \& Sons; 2005.

49. Zhang J, Liu H, Zhu K, Gong S, Dramsi S, Wang YT, Li J, Chen F, Zhang R, Zhou L, Lan L, Jiang $\mathrm{H}$, Schneewind O, Luo C, Yang CG. Antiinfective therapy with a small molecule inhibitor of Staphylococcus aureus sortase. Proc Natl Acad Sci U S A. 2014; 111:13517-22. [PubMed: 25197057]

50. Crouch SP, Kozlowski R, Slater KJ, Fletcher J. The use of ATP bioluminescence as a measure of cell proliferation and cytotoxicity. J Immunol Methods. 1993; 160:81-8. [PubMed: 7680699]

51. Lin JH, Perryman AL, Schames JR, McCammon JA. The relaxed complex method: Accommodating receptor flexibility for drug design with an improved scoring scheme. Biopolymers. 2003; 68:47-62. [PubMed: 12579579]

52. Amaro RE, Baron R, McCammon JA. An improved relaxed complex scheme for receptor flexibility in computer-aided drug design. J Comput Aided Mol Des. 2008; 22:693-705. [PubMed: 18196463] 
53. Marraffini LA, Dedent AC, Schneewind O. Sortases and the art of anchoring proteins to the envelopes of gram-positive bacteria. Microbiol Mol Biol Rev. 2006; 70:192-221. [PubMed: 16524923]

54. Falugi F, Kim HK, Missiakas DM, Schneewind O. Role of protein A in the evasion of host adaptive immune responses by Staphylococcus aureus. MBio. 2013; 4:e00575-13. [PubMed: 23982075]

55. Cascioferro S, Raffa D, Maggio B, Raimondi MV, Schillaci D, Daidone G. Sortase A Inhibitors: Recent Advances and Future Perspectives. J Med Chem. 2015; 58:9108-23. [PubMed: 26280844]

56. Muller K, Faeh C, Diederich F. Fluorine in pharmaceuticals: looking beyond intuition. Science. 2007; 317:1881-6. [PubMed: 17901324]

57. Mock M, Fouet A. Anthrax. Annu Rev Microbiol. 2001; 55:647-71. [PubMed: 11544370]

58. Scott CJ, McDowell A, Martin SL, Lynas JF, Vandenbroeck K, Walker B. Irreversible inhibition of the bacterial cysteine protease-transpeptidase sortase (SrtA) by substrate-derived affinity labels. Biochem J. 2002; 366:953-8. [PubMed: 12069686]

59. Oh KB, Nam KW, Ahn H, Shin J, Kim S, Mar W. Therapeutic effect of (Z)-3-(2,5dimethoxyphenyl)-2-(4-methoxyphenyl) acrylonitrile (DMMA) against Staphylococcus aureus infection in a murine model. Biochem Biophys Res Commun. 2010; 396:440-4. [PubMed: 20433810] 
A

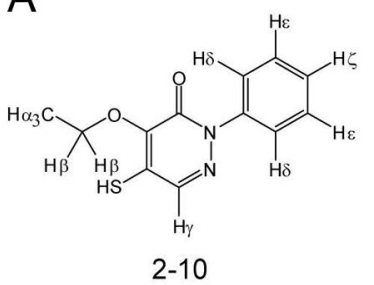

B
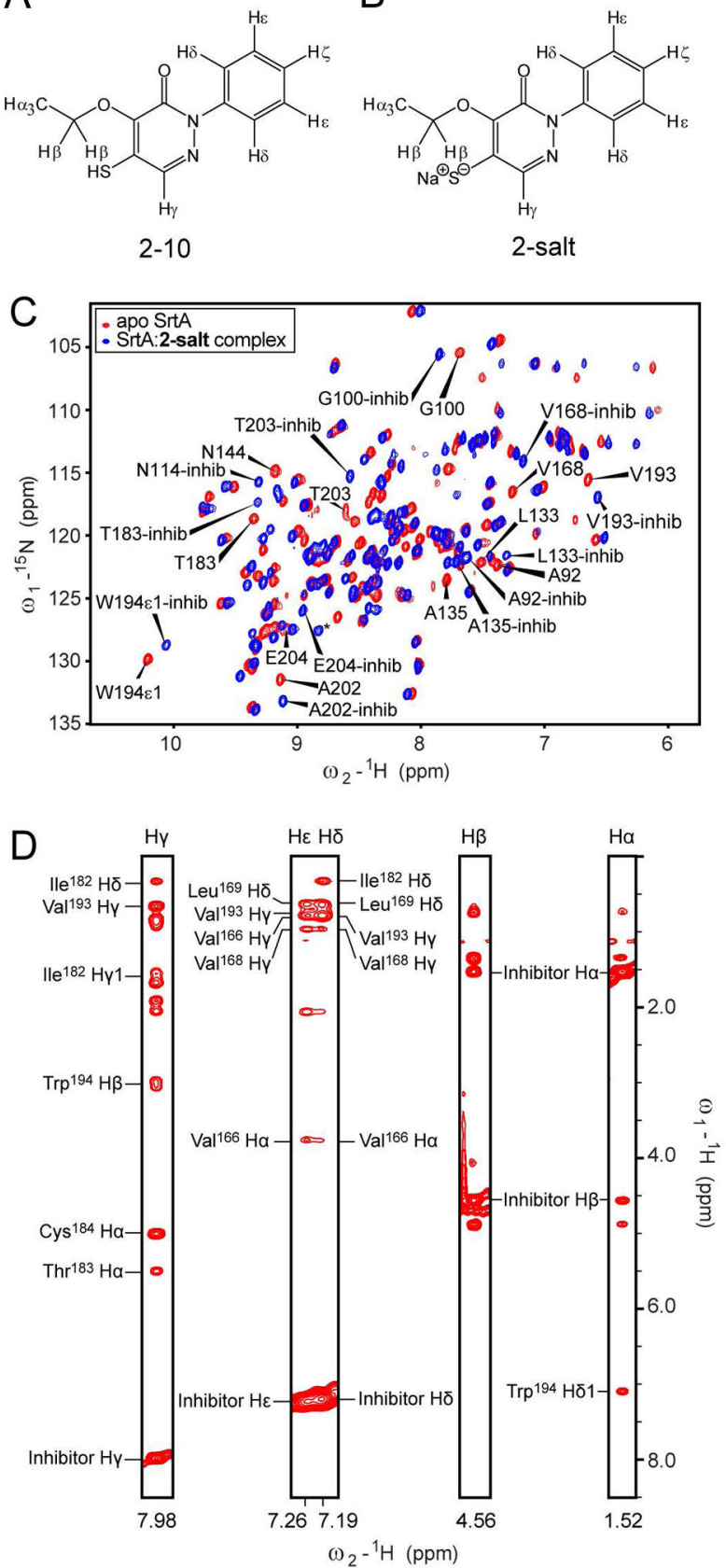

Figure 1.

NMR spectra of SrtA:2-salt complex. (A) Structure of compound 2-10 (4-ethoxy-5mercapto-2-phenyl-3(2H)-pyridazinone), a previously discovered Sa-SrtA inhibitor with $\mathrm{IC}_{50}$ of $13 \mu \mathrm{M}$ or $1.5 \mu \mathrm{M}$ when dimerized through disulfide bond. (B) Structure of the sodium thiolate version of 2-10 (2-salt) used in NMR and LC-MS studies. (C) ${ }^{1} \mathrm{H}_{-}{ }^{15} \mathrm{~N}$ HSQC of apo (red) and inhibitor bound (blue) SrtA after 24 hours of incubation. Peaks that had significant chemical shift changes $(\Delta \delta)$ upon binding of the inhibitor are labeled.

Significant chemical shift changes are defined as greater than the average $\Delta \delta+$ one standard 
deviation, where $\Delta \delta=\sqrt{\left(\Delta \delta_{H}\right)^{2}+\left(\Delta \delta_{N} / 6.49\right)^{2}}$, and $\Delta \delta_{H}$ and $\Delta \delta_{N}$ are the chemical shift changes in $\mathrm{Hz}$ for amide proton and amide nitrogen, respectively. (D) selected panels showing intermolecular NOEs between SrtA and 2-salt. Identity and chemical shifts for each inhibitor proton are shown at the top and bottom of each panel, respectively. Each assigned cross peak is labeled with the corresponding proximal SrtA proton. Each assignment was verified in a $3 \mathrm{D}^{13} \mathrm{C}$-editied NOESY by identifying a corresponding inhibitor cross peak. Not all cross peaks could be identified due to chemical shift ambiguities, including all NOESY cross peaks from the inhibitor $\mathrm{H} \beta$ protons. 

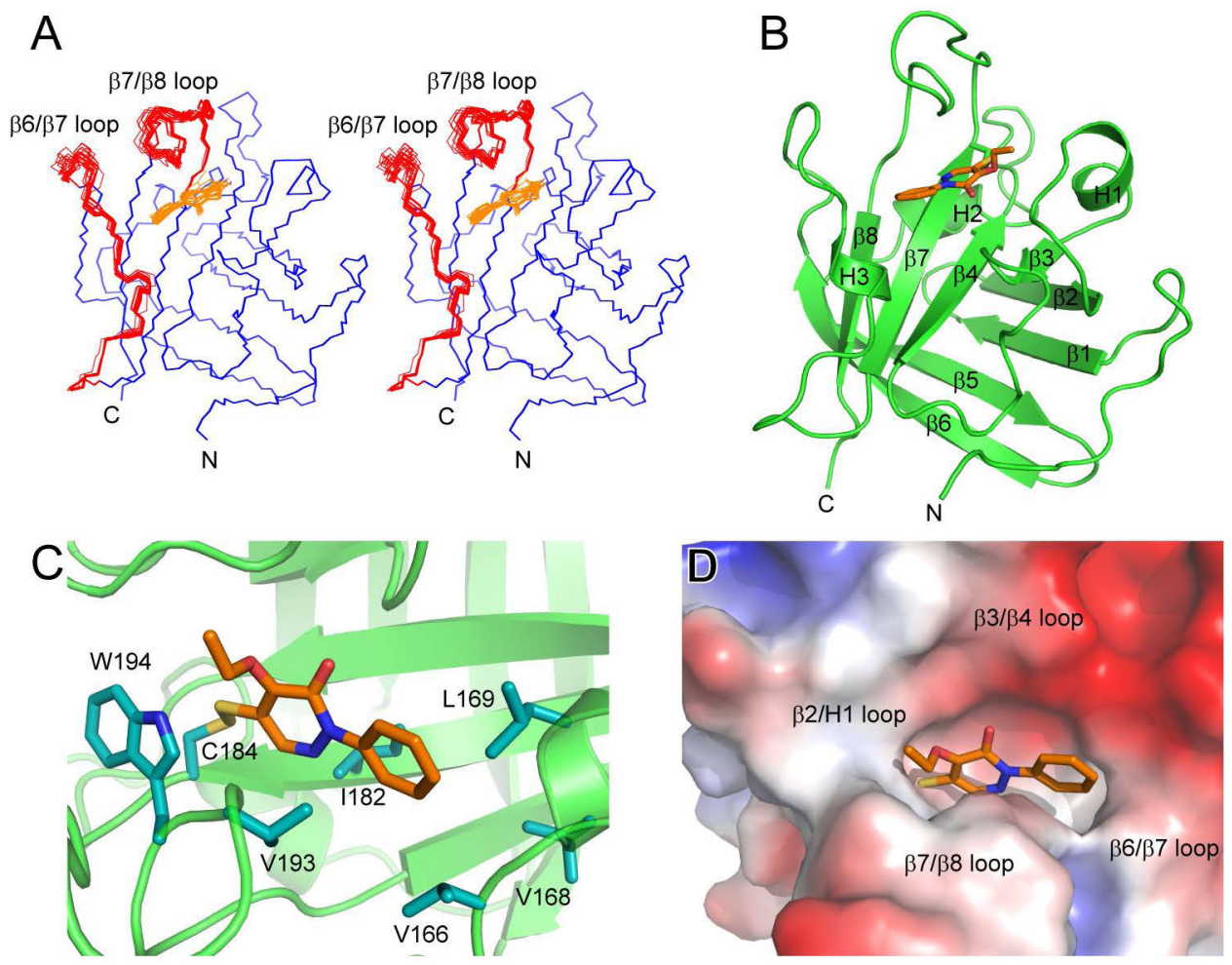

Figure 2.

NMR solution structure of the SrtA:2-salt complex. (A) Cross-eyed stereo image showing the ensemble of 20 lowest energy structures of the SrtA:2-salt complex. The bound compound 2-salt is shown in orange, and protein backbone atoms allowed to move during molecular dynamics simulations are shown in red (Val ${ }^{161}$-Lys $^{175}$ and $\mathrm{Thr}^{183}{ }^{18 y s}{ }^{196}$ ). The bundle is aligned using all of the protein backbone heavy atoms and the heavy atoms from the inhibitor. (B) Ribbon structure of the SrtA:2-salt complex. The covalently bound inhibitor is shown in stick representation and colored orange. (C) Expanded view of the SrtA active site with the inhibitor bound. Side chains of residues exhibiting intermolecular NOEs to the inhibitor are shown in cyan sticks. A hydrophobic pocket formed by $\mathrm{Val}^{166}, \mathrm{Val}^{168}$, Leu $^{169}$, and Ile ${ }^{182}$ bind the 2-phenyl group of the inhibitor, while the remainder of the inhibitor is positioned over the $\beta 7$ and $\beta 8$ strands. (D) Expanded view of the SrtA active site with the protein represented by its solvent accessible surface. The surface is colored by its electrostatic properties from acidic (red) to basic (blue). 

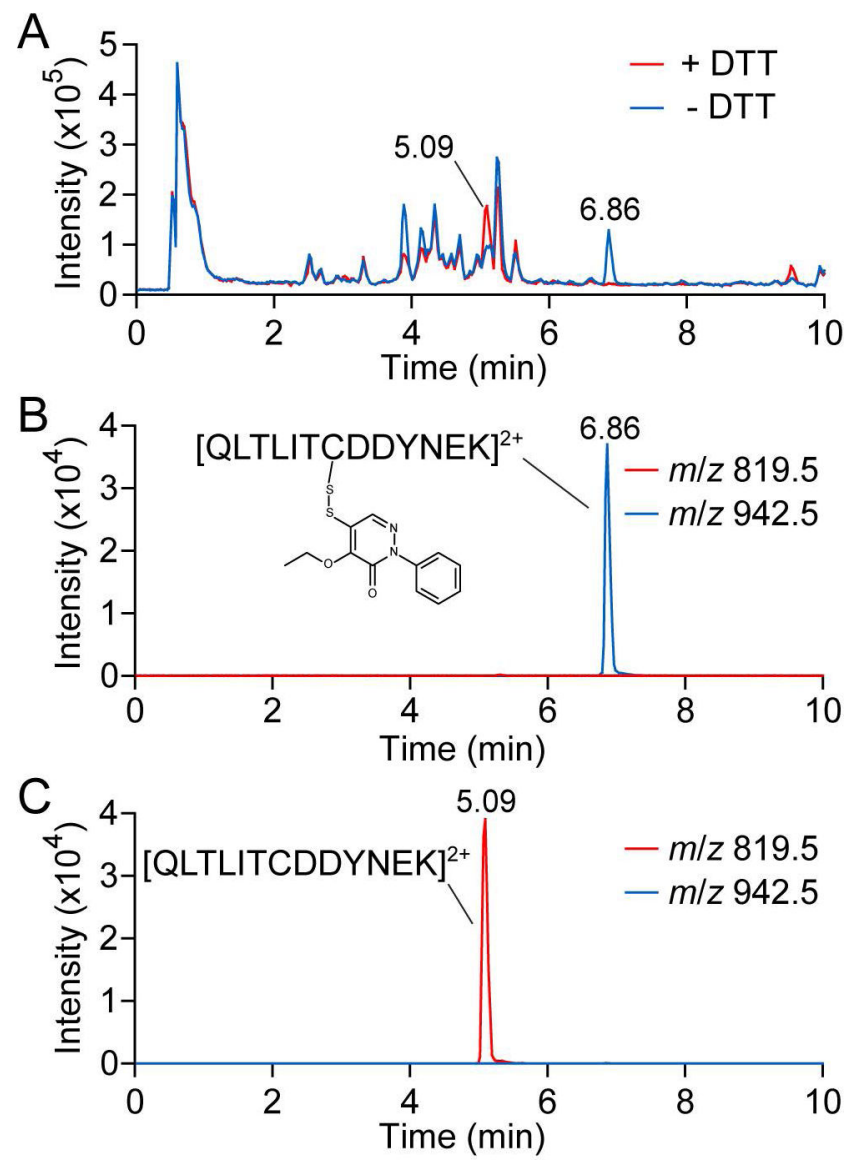

Figure 3.

LC-MS traces of the SrtA:2-salt complex. (A) Overlay of the total ion traces of trypsindigested 1:1 mixture of SrtA and 2-salt before (blue) and after (red) addition of 5 mM DTT. Major differences occur at retention times $5.09 \mathrm{~min}$ and $6.86 \mathrm{~min}$. (B) Chromatogram of trypsin-digested sample without addition of DTT. Only the traces of ions with $\mathrm{m} / \mathrm{z}$ of 819.5 (red) and 942.5 (blue) are shown, which correspond to the $[\mathrm{M}+2 \mathrm{H}]^{2+}$ ions of the unmodified Cys ${ }^{184}$-containing peptide and 2-salt modified version of the peptide, respectively. (C) Similar to (B), but the chromatogram shown is that of DTT added trypsin-digested sample. 


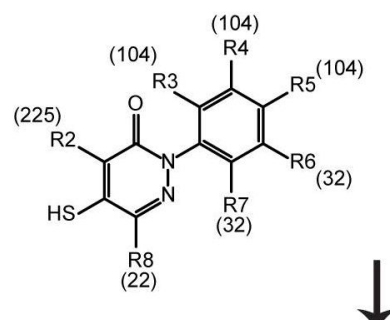

(12)

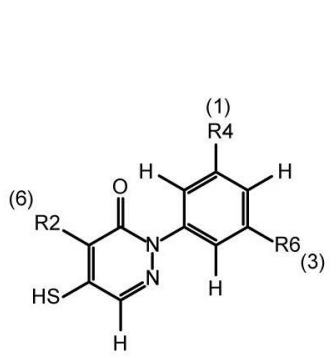

Docking: Stage 1

1. Generates 623 substituents at 7 sites with CombiGlide

2. Docks $\sim 6,000$ compounds to the NMR structure of SrtA-inhibitor complex with Glide

Docking: Stage 2

3. Identifies 4 favorable sites for modification and 22 substituents based on docking scores

4. Generates 432 compounds with all possible combinations of substituents with CombiGlide

5. Docks the compounds to the NMR and 25 representative MD simulated structures with Glide
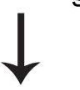

Experimental Testing

6 . Identifies the most favorable substituents at each site among the top $10 \%$ ligands

7. Selects 10 substituents at 3 sites for synthesis and experimental testing

Figure 4.

Overview of the molecular docking and experimental testing process. Description of the docking, substituents selection, and experimental testing processes are shown on the right. The structure of the core of the pyridazinone compound is shown on the left. The number of substituents at each site is indicated in parenthesis. 

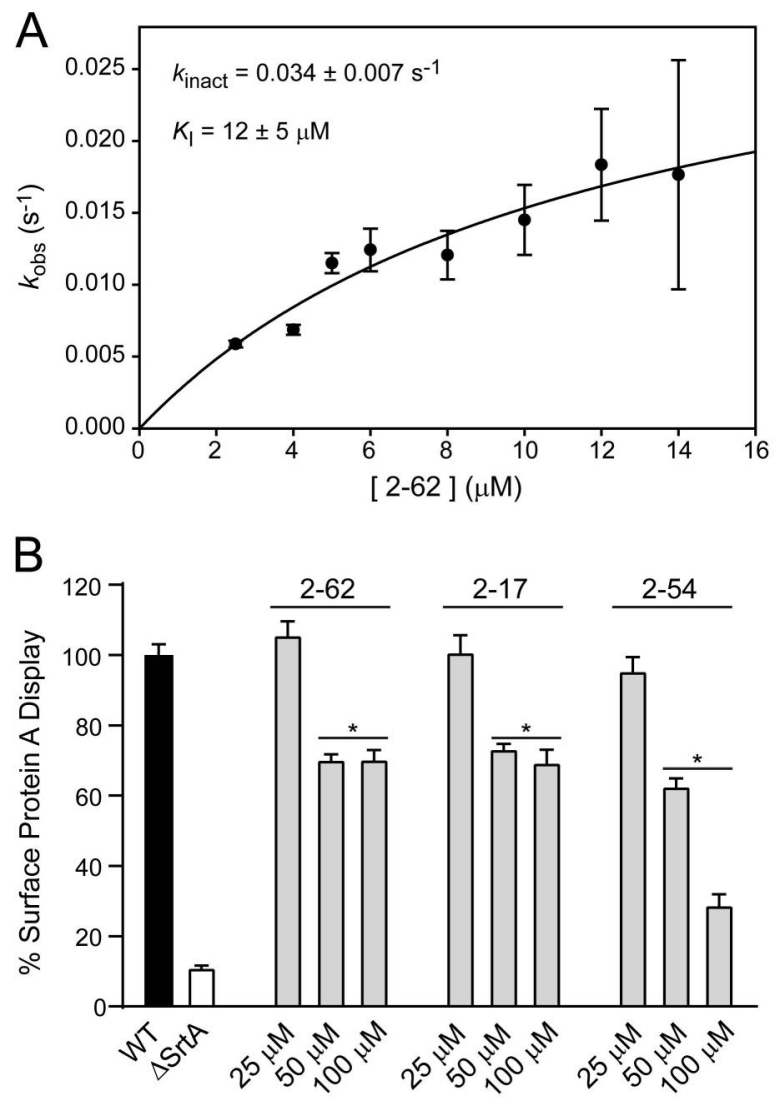

Figure 5.

Inhibitor inactivation kinetics and effect on protein display. (A) The rate of SrtA inhibition by compound 2-62 was determined by calculating $k_{\mathrm{obs}}$ for each inhibitor concentration using equation 3 and subsequently calculating $k_{\text {inact }}$ and $K_{\mathrm{I}}$ using equation 4 listed in Materials and Methods section. This graph shows a representative curve of fitting the $k_{\mathrm{obs}}$ data with equation 4. (B) The abundance of surface protein A displayed on $S$. aureus cell wall in the absence (control) or presence of pyridazinone inhibitor was quantified with a FITC-labeled $\mathrm{IgG}$ binding assay. Statistical significance $(* \mathrm{P}<0.0007)$ was determined using the unpaired, two-tailed Student t test $(\mathrm{n}=3$, shown with standard deviation). 


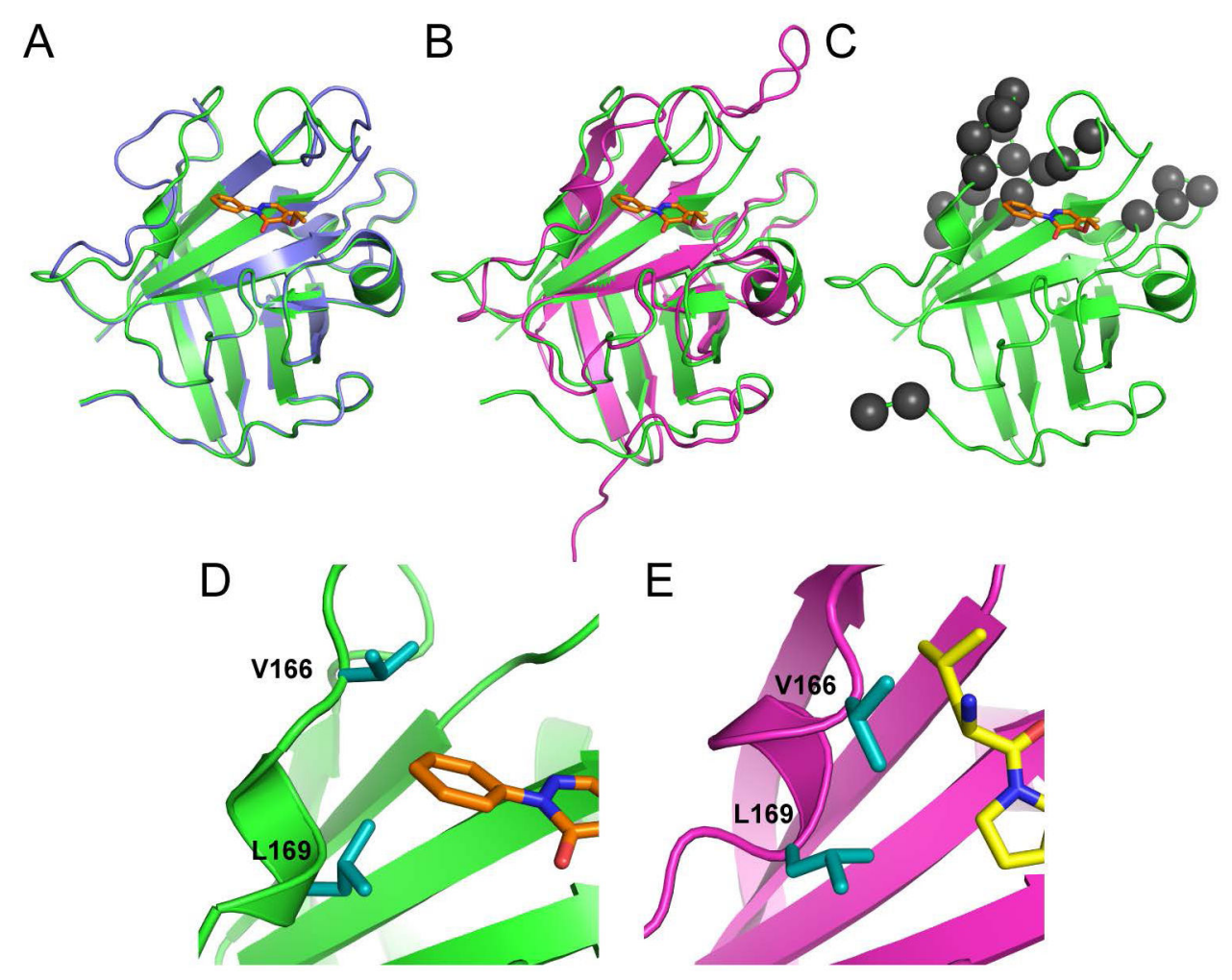

Figure 6.

Comparison of the SrtA:2-salt structure with apo- and holo-SrtA structures. SrtA:2-salt complex (green) superimposed with (A) apo-SrtA (blue, PDB code 1IJA) and (B) SrtA:LPAT* complex with the substrate analog LPAT* removed (pink, PDB code 2KID). In apo-SrtA, residues Ser ${ }^{157}$-Lys ${ }^{175}$ are unstructured in the $\beta 6 / \beta 7$ loop. In inhibitor complex, a portion of this loop is immobilized. The $\beta 6 / \beta 7$ loop in the SrtA:2-salt complex is unstructured from residues $\mathrm{Thr}^{156}$-Asp ${ }^{165}$, similar to apo-SrtA, and adopts a single conformation from residues Val ${ }^{166}$-Lys ${ }^{175}$, as seen in the SrtA:LPAT* ${ }^{*}$ structure. The $\beta 7 / \beta 8$ loop adopts a conformation more closely resembling the apo-SrtA structure. (C) Nonproline amide crosspeaks that were not detected in the ${ }^{1} \mathrm{H}_{-}{ }^{15} \mathrm{~N}$ HSQC spectra are shown in gray spheres. These residues include $\mathrm{Met}^{59}-\mathrm{Gln}^{60}, \mathrm{Thr}^{121}-\mathrm{Asp}^{124}, \mathrm{Lys}^{137}, \mathrm{Thr}^{156} \mathrm{VVal}^{166}$, and Lys ${ }^{196}-\mathrm{Phe}^{200}$. (D) Expanded view of the $\beta 6 / \beta 7$ loop $3_{10}$-helix in the SrtA:2-salt complex with 2-salt represented as orange sticks. (E), expanded view of the $\beta 6 / \beta 7$ loop and $3_{10}$-helix in the SrtA:LPAT* complex with LPAT* represented as yellow sticks. 

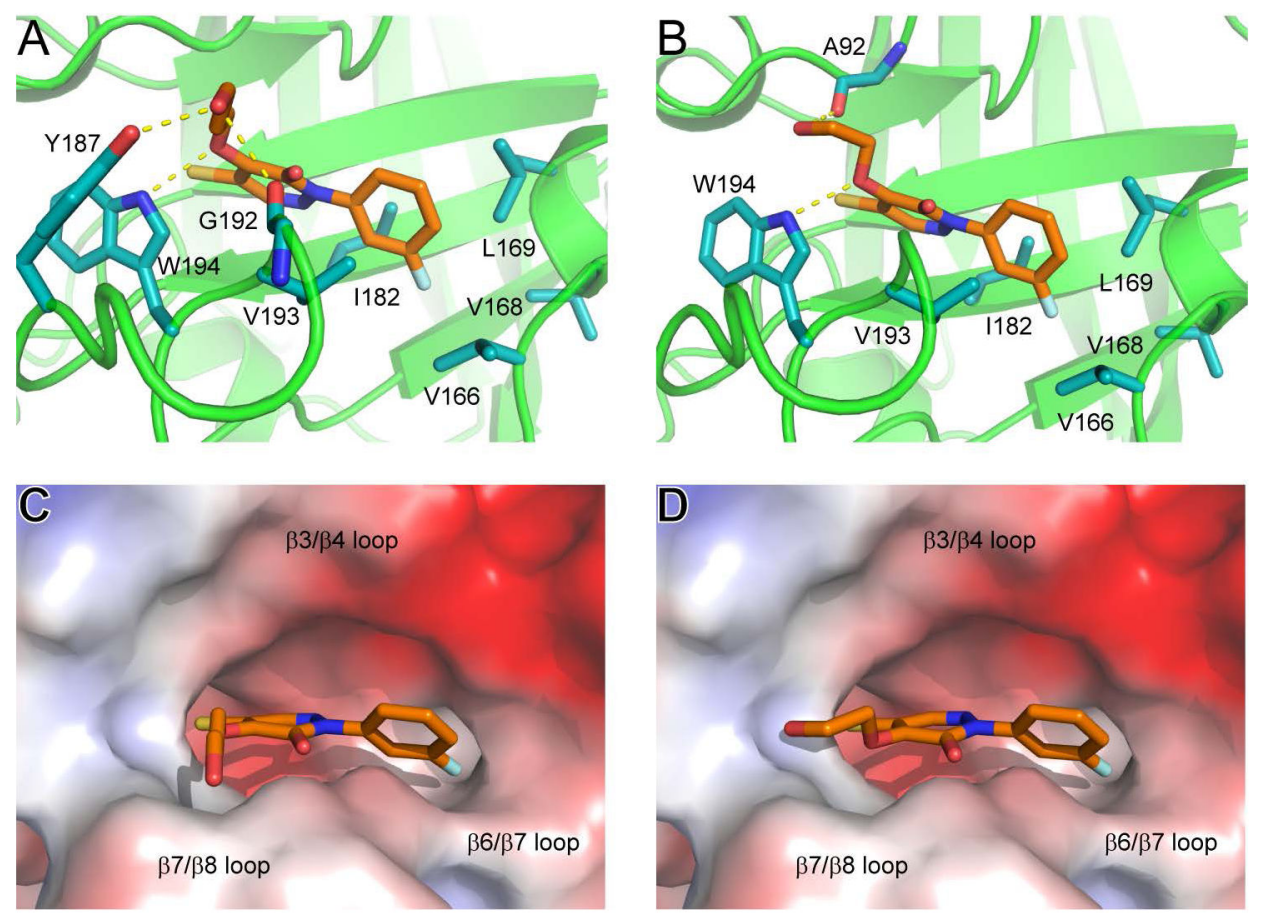

Figure 7.

Docking poses of compounds 2-61 and 2-62. Expanded view of the active site showing interactions between the protein and compound 2-62 (A, C) and compound 2-61 (B, D). In (A) and (B), residues that contact the compound are shown as cyan sticks. Hydrogen bonds are indicated by yellow dotted lines. In (C) and (D), the protein solvent accessible surface is shown and colored by its electrostatic properties from acidic (red) to basic (blue). 
Table 1

Statistics for the NMR modeled structure of SrtA bound to a pyridazinone inhibitor 2-salt

\begin{tabular}{|c|c|c|}
\hline & $\langle S A\rangle^{a}$ & $\langle\overline{S A}\rangle^{a}$ \\
\hline \multicolumn{3}{|l|}{ R.m.s. deviations from NOE interproton distance restraints $(\AA)$} \\
\hline Intramolecular $^{b}(156)$ & $0.075 \pm 0.003$ & 0.087 \\
\hline Intermolecular (20) & $0.104 \pm 0.007$ & 0.121 \\
\hline R.m.s. deviations from dihedral angle restraints (degrees) ${ }^{\mathcal{c}}(43)$ & $0.824 \pm 0.068$ & 0.715 \\
\hline R.m.s. deviations from ${ }^{3} J_{\mathrm{HN}}{ }^{a}$ coupling constants $(\mathrm{Hz})^{\mathcal{C}}(10)$ & $0.451 \pm 0.027$ & 0.772 \\
\hline \multicolumn{3}{|l|}{ Deviations from idealized covalent geometry } \\
\hline Bonds $(\AA)$ & $0.0040 \pm 0.0000$ & 0.00574 \\
\hline Angles (degrees) & $1.048 \pm 0.002$ & 1.0697 \\
\hline Impropers (degrees) & $1.545 \pm 0.026$ & 1.5550 \\
\hline \multicolumn{3}{|l|}{ PROCHECK-NMR $^{d}$} \\
\hline Most favorable region $(\%)$ & $66.5 \pm 2.9$ & 66.7 \\
\hline Additionally allowed region $(\%)$ & $29.3 \pm 3.2$ & 28.6 \\
\hline Generously allowed region (\%) & $4.3 \pm 1.5$ & 4.8 \\
\hline Disallowed region (\%) & $0.0 \pm 0.0$ & 0 \\
\hline \multicolumn{3}{|l|}{ Coordinate precision ${ }^{e}$} \\
\hline Protein backbone $(\AA)$ & $0.20 \pm 0.07$ & \\
\hline Protein heavy atoms $(\AA)$ & $0.69 \pm 0.06$ & \\
\hline
\end{tabular}

${ }^{a}$ The notation of the NMR structures is as follows: $\langle\boldsymbol{S A}\rangle$ represent an ensemble of 20 best structures calculated by simulated annealing. $\langle\overline{\boldsymbol{S A}}\rangle$ is the average energy-minimized structure. The number of terms for each restraint is given in parentheses. None of the structures exhibited distance violations greater than $0.5 \AA$, dihedral angle violations greater than $5^{\circ}$, or coupling constant violations greater than $2 \mathrm{~Hz}$.

$b_{\text {Intramolecular NOE distance restraints include any NOE signals from any residue in the Sa-SrtA enzyme to residues Val }}{ }^{166}$-Lys ${ }^{175}$, Thr ${ }^{183}$.

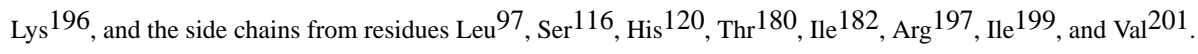

$c_{\text {Experimental backbone dihedral angle restraints comprised } 21 \mathrm{f} \text { and } 22 \psi \text { angles within residues Val }}{ }^{166}$-Lys $^{175}$ and Thr $^{183}$-Lys ${ }^{196}$.

$d_{\text {PROCHECK-NMR data includes residues Val }}{ }^{166}$-Lys 175 and Thr ${ }^{183}$-Lys 196 of the Sa-SrtA:inhibitor complex.

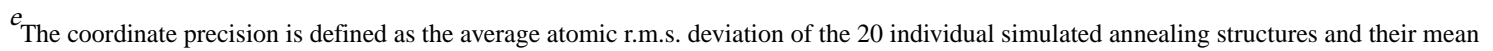
coordinates. The reported values are for residues Val ${ }^{166}$-Lys $175_{\text {and Thr }}{ }^{183}$-Lys 196 of the Sa-SrtA:inhibitor complex for the protein backbone r.m.s. deviation. In addition, residues $\mathrm{Leu}^{97}$, $\mathrm{Ser}^{116}$, $\mathrm{His}^{120}, \mathrm{Thr}^{180}, \mathrm{Ile}^{182}, \mathrm{Arg}^{197}, \mathrm{Ile}^{199}$, and $\mathrm{Val}^{201}$ were included for calculating protein heavy atom r.m.s. deviation. 

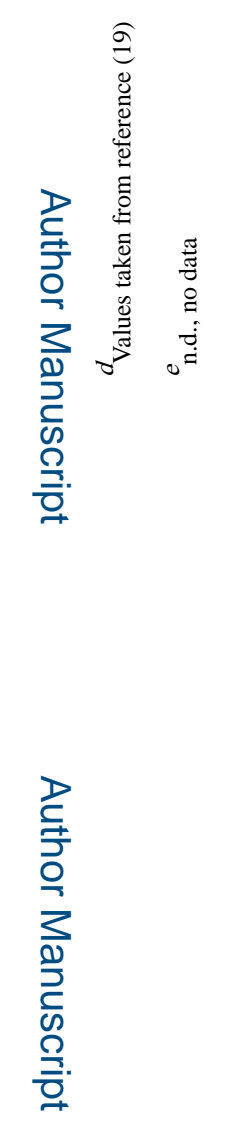

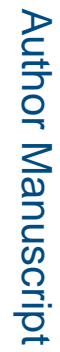

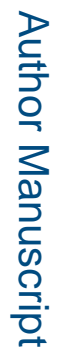

Chem Biol Drug Des. Author manuscript; available in PMC 2018 September 01. 
Table 3

Inactivation kinetics of pyridazinone compound derivatives

\begin{tabular}{cccc}
\hline Compound & $\boldsymbol{k}_{\text {inact }}\left(\mathbf{s}^{-\mathbf{1}}\right)$ & $\boldsymbol{K}_{\mathbf{I}}(\boldsymbol{\mu M})$ & $\boldsymbol{k}_{\text {inact }} / \boldsymbol{K}_{\mathbf{I}}\left(\boldsymbol{\mu \mathbf { M } ^ { - 1 } \mathbf { s } ^ { - \mathbf { 1 } } )}\right.$ \\
\hline $2-17$ & $0.004 \pm 0.001$ & $5 \pm 3$ & $0.0008 \pm 0.0005$ \\
$2-54$ & $0.015 \pm 0.003$ & $24 \pm 9$ & $0.0006 \pm 0.0003$ \\
$2-62$ & $0.034 \pm 0.007$ & $12 \pm 5$ & $0.003 \pm 0.001$ \\
\hline
\end{tabular}

